\title{
PAIR-DENSE RELATION ALGEBRAS
}

\author{
ROGER D. MADDUX
}

\begin{abstract}
The central result of this paper is that every pair-dense relation algebra is completely representable. A relation algebra is said to be pair-dense if every nonzero element below the identity contains a "pair". A pair is the relation algebraic analogue of a relation of the form $\{\langle a, a\rangle,\langle b, b\rangle\}$ (with $a=b$ allowed). In a simple pair-dense relation algebra, every pair is either a "point" (an algebraic analogue of $\{\langle a, a\rangle\}$ ) or a "twin" (a pair which contains no point). In fact, every simple pair-dense relation algebra $\mathfrak{A}$ is completely representable over a set $U$ iff $|U|=\kappa+2 \lambda$, where $\kappa$ is the number of points of $\mathfrak{A}$ and $\lambda$ is the number of twins of $\mathfrak{A}$.

A relation algebra is said to be point-dense if every nonzero element below the identity contains a point. In a point-dense relation algebra every pair is a point, so a simple point-dense relation algebra $\mathfrak{A}$ is completely representable over $U$ iff $|U|=\kappa$, where $\kappa$ is the number of points of $\mathfrak{A}$. This last result actually holds for semiassociative relation algebras, a class of algebras strictly containing the class of relation algebras. It follows that the relation algebra of all binary relations on a set $U$ may be characterized as a simple complete point-dense semiassociative relation algebra whose set of points has the same cardinality as $U$.

Semiassociative relation algebras may not be associative, so the equation $(x ; y) ; z=x ;(y ; z)$ may fail, but it does hold if any one of $x, y$, or $z$ is 1. In fact, any rearrangement of parentheses is possible in a term of the form $x_{0} ; \ldots ; x_{\alpha-1}$, in case one of the $x_{\kappa}$ 's is 1 . This result is proved in a general setting for a special class of groupoids.
\end{abstract}

\section{INTRODUCTION}

In its most basic form, a representation result for relation algebras is simply a theorem which asserts that every relation algebra having a certain property is representable. This paper presents several new theorems of this kind. The importance of such results stems from the fact that not all relation algebras are representable. Until Lyndon's counterexample in [L50], one could have hoped (as in [T41, pp. 87-88]) that the ultimate representation result would be true, namely that every relation algebra would be representable. This happy situation exists for groups and Boolean algebras. The Stone Representation Theorem implies that every Boolean algebra is isomorphic to a Boolean algebra of sets, i.e.,

Received by the editors November 3, 1988.

1980 Mathematics Subject Classification (1985 Revision). Primary 03G15, 03G25; Secondary 06A23, 06E10, $20 \mathrm{~L} 05$.

Key words and phrases. Relation algebra, semiassociative relation algebra, representable, completely representable, associativity, groupoid. 
an algebra whose elements are sets and whose operations are the standard settheoretic operations of intersection, union, and complementation with respect to the union of all the sets in the algebra. Similarly, the Cayley Representation Theorem implies that every group $\mathfrak{G}$ is isomorphic to a group of permutations, i.e., a group whose elements are permutations and whose operations are the standard ones which gave rise to the group concept, namely, the composition of permutations, the operation of forming the inverse of a permutation, and the distinguished identity permutation which maps every element to itself.

The relation algebras which correspond to Boolean algebras of sets and to groups of permutations are called proper relation algebras. The universe $A$ of a proper relation algebra $\mathfrak{A}$ is a nonempty family of binary relations between elements of some set $U$. ( $U$ may be empty.) The fundamental operations of $\mathfrak{A}$ are certain natural set-theoretic operations on binary relations, under which $A$ is closed. In fact, $\mathfrak{A}$ is a Boolean algebra, with the operations of union, intersection, and complementation with respect to the union of the relations in $A$ ( $A$ may not coincide with $U \times U)$, together with the binary operation of composition, the unary operation of conversion, and the identity relation on $U$, namely $\operatorname{Id}_{U}=\{\langle x, x\rangle: x \in U\}$. Composition, which was introduced by Augustus De Morgan ([D1856] and especially [D1864]; see [D66, pp. 55-57, 208,221 , etc.]), is defined as follows for any two binary relations $R$ and $S$ :

$$
R \mid S=\{\langle x, z\rangle: \text { for some } y,\langle x, y\rangle \in R \text { and }\langle y, z\rangle \in S\} .
$$

(The notation "|" was used by Russell and Whitehead [WR10]. De Morgan just used juxtaposition, as did Peirce [P33]. Schröder [S1895] used “;”.) Conversion, also introduced by De Morgan ([D1864]; see [D66, p. 222]), is defined for a relation $R$ as follows:

$$
R^{-1}=\{\langle y, x\rangle:\langle x, y\rangle \in R\} .
$$

A relation algebra is an algebra of the form

$$
\mathfrak{A}=\left\langle A,+, \cdot,^{-}, 0,1, ;,^{\smile}, 1^{\prime}\right\rangle,
$$

where $\mathfrak{A}=\langle A,+, \cdot,-, 0,1\rangle$ is a Boolean algebra, ; is an associative binary operation (corresponding to composition $\mid$ ), $>$ is a unary operation (corresponding to conversion ${ }^{-1}$ ), $1^{\prime}$ is an identity element for ; (i.e., $\left.1^{\prime} ; x=x=x ; 1^{\prime}\right)$, and De Morgan's "Theorem K" [D1864] holds: if $x ; y \leq z$ then $\breve{x} ; \bar{z} \leq \bar{y}$ and $\bar{z} ; \breve{y} \leq \bar{x}$. Of course, every proper relation algebra is indeed a relation algebra. A relation algebra $\mathfrak{A}$ which is isomorphic to a proper relation algebra is said to be representable, and an isomorphism from $\mathfrak{A}$ to a proper relation algebra is called a representation of $\mathfrak{A}$. A representation is complete if it preserves all meets and joins, and a relation algebra is completely representable if it has a complete representation.

A fundamental example of a Boolean algebra is the algebra of all subsets of a nonempty set $U$. Similarly, a fundamental example of a group is the group 
of all permutations of $U$. For relation algebras an analogous example is $\mathfrak{R e} U$, the algebra of all binary relations on $U$. It turns out that $\mathfrak{R e} U$ has a peculiarity not shared by the other two examples: it is simple (has at least two elements and no nontrivial homomorphic images). Another fundamental example in the theory of relation algebras is the algebra $\mathfrak{S b} E$ of all relations contained in a given equivalence relation $E . \mathfrak{S b} E$ is isomorphic to the direct product of the algebras $\mathfrak{R e} U$, where $U$ ranges over the equivalence classes of $E$. A relation algebra is proper just in case it is isomorphic to a subalgebra of some $\mathfrak{S b} E$.

The arithmetic of relation algebras is a kind of mixture of Boolean algebra and group identities. Part of the reason for this is obvious: a relation algebra is a Boolean algebra with additional operators. Another reason is that every group $\mathfrak{G}=\left\langle G, \cdot,^{-1}, e\right\rangle$ gives rise to a naturally correlated relation algebra $\mathfrak{C m} \mathfrak{G}$, called the complex, or Frobenius, algebra of $\mathfrak{G}$. (See [JT48]; the observation that Frobenius algebras are relation algebras was first made by J. C. C. McKinsey.) The complex algebra $\mathfrak{C m} \mathfrak{G}$ is the Boolean algebra of all subsets of $G$ together with the multiplication and inversion of "complexes" (subsets of $G)$, and the singleton complex $\{e\}$. Thus

$$
\mathfrak{C m} G=\left\langle\mathrm{Sb}(G), \cup, \cap,-, \varnothing, G, ;,{ }^{\smile},\{e\}\right\rangle
$$

where, for $X, Y \subseteq G, X ; Y=\{x y: x \in X, y \in Y\}$ and $X^{\smile}=\left\{x^{-1}: x \in X\right\}$.

It is easy to prove that the complex algebra of a group $\mathfrak{G}$ is a representable relation algebra. By Cayley's theorem, $\mathfrak{G}$ is isomorphic to a group of permutations of some set $U$, so every element of $\mathfrak{G}$ is correlated with a binary relation on $U$ (which happens to be a permutation). Then, for each subset $X$ of $G$, let $R(X)$ be the union of the relations correlated with the elements of $X$. It is easy to show that $R$ is a representation of $\mathfrak{C m} \mathfrak{G}$. This proves an example of the simplest kind of representation result: if $\mathfrak{A}$ is a complex algebra of a group, then $\mathfrak{A}$ is representable. More powerful representation results may say something more about the types of representations that $\mathfrak{A}$ can have. For example, $R$ embeds $\mathfrak{C m} \mathfrak{G}$ into $\mathfrak{R e} U=\mathfrak{S b}(U \times U)$, so we know that $\mathfrak{C m} \mathfrak{G}$ is not just representable, but that $\mathfrak{C m} \mathfrak{G}$ has an embedding into $\mathfrak{S b} E$ for a rather special kind of equivalence relation $E$, namely one with just one equivalence class. By Theorem 4.26 of [JT52], such an embedding exists for a representable relation algebra just in case that algebra is simple. (Of course, $\mathfrak{C m} \mathfrak{G}$ is simple.) This example is typical; the conclusions of a representation result can often be considerably strengthened if simplicity is added to the hypotheses.

The representation $R$ has another special property. It is complete, that is, it preserves all infinite meets and joins. Every representation of a finite representable relation algebra is trivially complete, but there are representable relation algebras which have no complete representations. A relation algebra with a complete representation is said to be completely representable. Thus $\mathfrak{C m} \mathfrak{G}$ is completely representable for every group $\mathfrak{G}$.

The main representation results of this paper use the concepts of pair-density and point-density, which are defined in terms of certain kinds of elements called 
points, pairs, and twins. Let $\mathfrak{A}$ be a relation algebra, and let $x$ be a nonzero element of $\mathfrak{A}$. We say $x$ is a point if $x ; 1 ; x \leq 1$, and $x$ is a pair if $x ; 0^{\prime} ; x ; 0^{\prime} ; x \leq 1^{\prime}$, where $0^{\prime}=1^{\prime-}$. A $t$ win is a pair which does not contain a point.

To understand what these conditions mean, suppose $\mathfrak{A}$ is a subalgebra of $\mathfrak{R e}$. By deciphering the definitions of point and pair we find that $x$ is a point just in case $x=\{\langle a, a\rangle\}$ for some $a \in U$, and $x$ is a pair just in case there are $a, b \in U$, which need not be distinct, such that $x=\{\langle a, a\rangle,\langle b, b\rangle\}$. Consequently, $x$ is a twin just in case there are distinct $a, b \in U$ such that $x=\{\langle a, a\rangle,\langle b, b\rangle\}$, and neither $\{\langle a, a\rangle\}$ nor $\{\langle b, b\rangle\}$ belongs to $\mathfrak{A}$.

Clearly, for subalgebras of $\mathfrak{R e} U$, every point is both a pair and an atom, every twin is also a pair and an atom, no twin is a point, the join of two distinct points is a pair, and every pair is either a twin or a point or the join of two points. All these properties can be proved for an arbitrary simple relation algebra $\mathfrak{A}$. ( $\mathfrak{R e} U$ is simple, and simplicity is essential in the proofs.)

We call a relation algebra $\mathfrak{A}$ pair-dense if every nonzero element below 1 ' contains a pair, and point-dense if every nonzero element below 1' contains a point. Letting $\operatorname{Pr} \mathfrak{A}$ and $\operatorname{Pt} \mathfrak{A}$ be the sets of pairs and points of $\mathfrak{A}$, respectively, we conclude that $\mathfrak{A}$ is pair-dense iff $\sum \operatorname{Pr} \mathfrak{A}=1$ ', and point-dense iff $\sum \operatorname{Pt} \mathfrak{A}=$ 1 '.

Since every point is a pair, it follows that point-density implies pair-density. The converse is false. To get an example showing this, we need a relation algebra which has pairs, but not points, below every identity element. Hence we need an algebra with twins. Now $\mathfrak{R e} U$ is point-dense, but $\mathfrak{R e} U$ has no twins, so we must consider proper subalgebras of $\mathfrak{R e} U$. Consider the case $U=2=$ $\{0,1\}$. (Each natural number is the set of smaller natural numbers, i.e., $n=$ $\{0, \ldots, n-1\}.) \mathfrak{R e} 2$ has a subalgebra $\mathfrak{M}_{2}$ with universe $\left\{\varnothing, \operatorname{Id}_{2}, \operatorname{Di}_{2}, 2 \times 2\right\}$, where $\mathrm{Di}_{2}=\{\langle 0,1\rangle,\langle 1,0\rangle\} \cdot \mathfrak{M}_{2}$ has no points and exactly one twin, namely $\mathrm{Id}_{2}$. So $\mathfrak{M}_{2}$ is pair-dense but not point-dense.

The main results concerning pair-density are as follows. Suppose $\mathfrak{A}$ is a pairdense relation algebra. Then $\mathfrak{A}$ is completely representable. If $\mathfrak{A}$ is also simple, then $\mathfrak{A}$ is atomic, and $\mathfrak{A}$ is completely representable over a set $U$ just in case $|U|=|\operatorname{Pt} \mathfrak{A}|+2|\operatorname{Tw} \mathfrak{A}|$, where Tw $\mathfrak{A}$ is the set of twins of $\mathfrak{A}$. These results imply that every simple complete pair-dense relation algebra is isomorphic to one which is constructed in the following way.

Let $U$ be a set which is partitioned into one-element sets and two-element sets. Let $P$ be all the one-element sets in the partition, and let $T$ be all the two-element sets. Let $\sim$ be an equivalence relation on $T$. We get a simple complete atomic pair-dense relation algebra $\mathfrak{A} \subseteq \mathfrak{R e} U$ by specifying the atoms of $\mathfrak{A}$ as follows:

(1) if $\{a\} \in P$ then $\{\langle a, a\rangle\}$ is an atom,

(2) if $\{a, b\} \in T$ then $\{\langle a, a\rangle,\langle b, b\rangle\}$ and $\{\langle a, b\rangle,\langle b, a\rangle\}$ are atoms,

(3) if $\{a\},\{b\} \in P$ then $\{\langle a, b\rangle\}$ and $\{\langle b, a\rangle\}$ are atoms, 
(4) if $\{a, b\} \in T$ and $\{c\} \in P$ then $\{\langle a, c\rangle,\langle b, c\rangle\}$ and its converse $\{\langle c, a\rangle,\langle c, b\rangle\}$ are atoms,

(5) if $\{a, b\},\{c, d\} \in T$ and $\{a, b\} \sim\{c, d\}$ then $\{\langle a, c\rangle,\langle b, d\rangle\}$, $\{\langle a, d\rangle,\langle b, c\rangle\}$, and their converses $\{\langle c, a\rangle,\langle d, b\rangle\},\{\langle d, a\rangle,\langle c, b\rangle\}$ are atoms,

(6) if $\{a, b\},\{c, d\} \in T$ and $\{a, b\} \nsim\{c, d\}$ then $\{\langle a, c\rangle,\langle b, d\rangle$, $\langle a, d\rangle,\langle b, c\rangle\}$, and its converse $\{\langle c, a\rangle,\langle d, b\rangle,\langle d, a\rangle,\langle c, b\rangle\}$ are atoms.

The elements of $\mathfrak{A}$ are arbitrary unions of the atoms of $\mathfrak{A}$. The points of $\mathfrak{A}$ are the atoms $\{\langle a, a\rangle\}$ with $\{a\} \in P$, and the twins of $\mathfrak{A}$ are the atoms $\{\langle a, a\rangle,\langle b, b\rangle\}$ with $\{a, b\} \in T$. Suppose $\mathfrak{B} \subseteq \mathfrak{A}$ and $\mathfrak{B}$ contains all the atoms of $\mathfrak{A}$. Then $\mathfrak{B}$ is a simple pair-dense relation algebra, but $\mathfrak{B}$ need not be complete. The smallest example of such an algebra is the one whose elements are finite joins of atoms or the complements of such joins. Every simple pairdense relation algebra is isomorphic to such a $\mathfrak{B}$. Pair-dense relation algebras which are not simple cannot be so easily characterized, since pair-density is not preserved by homomorphisms, as shown by an example near the end of the paper.

The reasons for suspecting that pair-density implies representability are as follows. Relation algebraic equations correspond to first order sentences in which no more than three different variables occur [TG87]. It is possible to assert, with only three variables, that there are no more than two elements, but the assertion that there are no more than three elements requires four variables. Thus relation algebraic equations can handle and distinguish sets up to cardinality 2 , but for cardinality 3 or more all control is lost. (There is no equationally definable "triplet-density".) The equations which are true in all relation algebras correspond to those logically valid first order sentences in which at most three variables occur and which have proofs in which no sentence contains more than four distinct variables [Ma78a, Ma83, Ma89]. Binary relations between two two-element sets involve at most four elements, as shown in the construction above. If a relation algebra has a property which says, roughly speaking, that the algebra is built up from sets of cardinality 2 or less, then that sentence should imply representability. This is indeed the case for pair-density.

The representability of pair-dense relation algebras was inspired by a problem in a letter of August 1985 from H. Andréka and I. Németi. They asked for the construction of a certain sequence of nonrepresentable cylindric algebras of dimension 3. According to their problem, these algebras should be neatly embeddable in cylindric algebras of certain higher dimensions, and they should have some elements whose join is 1 and which have a property roughly corresponding to the equation $x ; 0^{\prime} ; x ; 0^{\prime} ; x \leq 1^{\prime}$. It seemed that cylindric algebras with all the properties and elements they wanted would have to be representable. For relation algebras this turned out to be the case. Because of the connections between cylindric algebras and relation algebras developed in [Ma78a, Ma89], 
this outcome suggests that the algebras they originally sought cannot be found.

A relation algebra is point-dense if it is pair-dense and every pair is a point (i.e., it has no twins). Consequently, every point-dense relation algebra $\mathfrak{A}$ is completely representable, and if $\mathfrak{A}$ is also simple, then $\mathfrak{A}$ is atomic and $\mathfrak{A}$ is completely representable over a set $U$ just in case $|U|=|\operatorname{Pt} \mathfrak{A}|$. It turns out, however, that these results continue to hold if $\mathfrak{A}$ belongs to a strictly larger class of algebras than relation algebras, namely, the class of semiassociative relation algebras.

Every relation algebra is associative, i.e., satisfies the identity $(x ; y) ; z=$ $x ;(y ; z)$. But a semiassociative relation algebra need not be associative. It is only required to satisfy a special case of the associative law, namely $(x ; 1) ; 1=$ $x ;(1 ; 1)$. The class of semiassociative relation algebras has special significance for algebraic logic, since it is the algebraic equivalent of first order logic with binary relation symbols and exactly three individual variables [Ma83, Ma89, TG87]. We will show that every point-dense semiassociative relation algebra is a relation algebra, and will use this fact to obtain results about point-dense semiassociative relation algebras as corollaries of results concerning pair-dense relation algebras.

So far we have encountered two new representation results of the simplest kind, namely

(A) every pair-dense relation algebra is representable,

(B) every point-dense semiassociative relation algebra is representable.

Since every relation algebra is a semiassociative relation algebra and pointdensity implies pair-density, these two theorems have an obvious common corollary:

(C) every point-dense relation algebra is representable.

They also have an obvious common generalization:

every pair-dense semiassociative relation algebra is representable.

But this is false. In the proof of Theorem 43(ii) there is an example of a semiassociative relation algebra which is pair-dense but is not a relation algebra. The example can be obtained by starting with the subalgebra of $\mathfrak{R e} 4$ generated by $\{\langle 0,0\rangle,\langle 1,1\rangle\}$, and then "splitting atoms".

Pair-density and point-density are such strong properties that representation results which use them as hypotheses apply only to a limited range of algebras. (On the other hand, far more can be proved for pair-dense and point-dense algebras than mere representability, as we shall see.) For example, $\mathfrak{G}$ is a group, then $\mathfrak{C} \mathfrak{G}$ is pair-dense if $\mathfrak{G}$ has only one element. Thus the representability of complex algebras of groups cannot be proved from the fact that every pair-dense relation algebra is representable. However, the representability of complex algebras of groups is an immediate consequence of the following corollary of Theorem 4.31 in [JT52].

(D) If $\mathfrak{A}$ is an atomic relation algebra in which every atom is functional, then $\mathfrak{A}$ is representable. 
An element $x$ of $\mathfrak{A}$ is functional if $\breve{x} ; x \leq 1$ '. Fn $\mathfrak{A}$ is the set of functional elements of $\mathfrak{A}$. An element of $\mathfrak{S b} E$ is functional iff it is a function. Complex algebras of groups are atomic and all their atoms are functional, so they are representable by $(\mathrm{D})$.

If $\mathfrak{A}$ is an atomic relation algebra with functional atoms, then $\sum F n \mathfrak{A}=$ 1. The latter condition is sufficient for representability, even if the algebra is atomless, as was shown in [MT76].

(E) If $\mathfrak{A}$ is a relation algebra in which $\sum F \mathfrak{A} \mathfrak{A}=1$, then $\mathfrak{A}$ is representable.

Theorem $(C)$ can be proved from (E). Suppose $\mathfrak{A}$ is a point-dense relation algebra. Assume $0 \neq x \in A$. Then $0 \neq 1^{\prime} \cdot(\breve{x} ; x)$. By point-density, there is some $y \in \operatorname{Pt} \mathfrak{A}$ such that $y \leq 1^{\prime} \cdot(\breve{x} ; x)$. Then $0 \neq y=1^{\prime} \cdot(\breve{x} ; x) \cdot y=$ $\left(1^{\prime} \cdot(\breve{x} ; x)\right) ; y \leq 1 ; x ; y$, so $x ; y \neq 0$, and $(x ; y)^{\smile} ;(x ; y)=y ; \breve{x} ; x ; y \leq$ $y ; 1 ; y \leq 1$ ', so $x ; y$ is functional. Thus every nonzero element of $\mathfrak{A}$ contains a nonzero functional element, i.e., $\sum F n \mathfrak{A}=1$, so $\mathfrak{A}$ is representable by (E). (One could also use (D) in place of (E), but this would require the additional work of showing that every simple point-dense relation algebra is atomic.)

A very important and early representation result is due to Tarski [T53; TG87, Theorem 8.4(iii)]:

(F) If $\mathfrak{A}$ is a relation algebra and $\breve{x} ; y=1$ for some $x, y \in$ Fn $\mathfrak{A}$, then $\mathfrak{A}$ is representable.

The statement of $(F)$ is actually equivalent to the semantical completeness of the formal system $\mathfrak{L}^{\times}$of [TG87], relative to sentences asserting the existence of "quasi-projections". (See the footnote, [TG87, p. 242].) This relative completeness of $\mathfrak{L}^{\times}$implies that almost all set theories can be formalized in $\mathfrak{L}^{\times}$

The next representation result, from [Ma78, Ma78a], is a direct generalization of both $(\mathrm{F})$ and $(\mathrm{E})$.

(G) If $\mathfrak{A}$ is a relation algebra and $\sum\{\breve{x} ; y: x, y \in \mathrm{Fn} \mathfrak{A}\}=1$, then $\mathfrak{A}$ is representable.

It is obvious that $(G)$ generalizes $(F)$. To see that $(G)$ generalizes $(E)$ one need merely note that $1^{\prime}$ is a functional element, $1^{\prime \prime}=1^{\prime}$, and $1^{\prime} ; x=x$.

Thus we have a sequence of ever more powerful representation results: $(\mathrm{G})$ implies (F) and (E), (E) implies (D), (D) implies (C), and (C) implies (B) relative to the result that every point-dense semiassociative relation algebra is a relation algebra. However, (A) stands outside this sequence, and thus is not a consequence of $(G)$. It is easy to construct arbitrarily large pair-dense relation algebras which do not satisfy the hypothesis of $(G)$. The construction given above yields such an algebra whenever $T \neq \varnothing$.

Incidentally, (D)-(G) all fail for semiassociative relation algebras. A single finite example suffices to show this for (D), (E), and (G). (See [Ma78a, Example 3(6)(i)(c), p. 64, and pp. 124-125].) No finite example can be used for (F), 
since every nontrivial semiassociative relation algebra with a pair of conjugated quasiprojections is infinite. One might expect this difference from the following observation: $\mathfrak{R e} U$ satisfies the hypotheses of $(\mathrm{F})$ only if $U$ is infinite, but $\mathfrak{R e} U$ always satisfies the hypotheses of (D), (E), and $(\mathrm{G})$, even if $U$ is finite. An infinite example showing that $(F)$ fails for semiassociative relation algebras was constructed by I. Németi. (See [N86, II.8(ii) and Theorem 17(viii)], or [N85, Theorem 3.7].)

The fact that $(C)$ can be generalized to $(B)$ suggests an investigation of the extent to which results for relation algebras hold for semiassociative relation algebras. More specifically, how much can the associative law be weakened until the result in question is no longer provable? For some results the associative law is not needed at all. The class of nonassociative relation algebras was therefore introduced in [Ma78a, Ma82] just to have a base for this kind of study. The definition of this class of algebras is obtained from the definition of relation algebras by simply deleting the associative law. It turns out that many (perhaps 80\%) of the arithmetical results in [CT51] were proved there, or could be proved, without any use of associativity. Perhaps another $10 \%$ of those results require only the semiassociative law, and thus hold for all semiassociative relation algebras. The remaining $10 \%$ require the associative law and fail in some semiassociative relation algebra. The class of all weakly associative relation algebras was introduced in [Ma82] for similar reasons. It is the class of all nonassociative relation algebras which satisfy the weak associative law: $\left(\left(1^{\prime} \cdot x\right) ; 1\right) ; 1=\left(1^{\prime} \cdot x\right) ;(1 ; 1)$. It turns out that this class of algebras is exactly the class for which the relative representation theorem holds (Theorem 5.20 of [Ma82]). Other results concerning atom structures and complex algebras also find their natural place in this class, i.e., they hold for all weakly associative relation algebras but not for all nonassociative relation algebras.

The four classes we have mentioned so far are varieties. (A variety is a class of algebras axiomatized by a set of identities (universally quantified equations).) In fact, they form a strictly increasing sequence of varieties: relation algebras, semiassociative relation algebras, weakly associative relation algebras, and nonassociative relation algebras. Every result in this paper is stated and proved for the largest possible variety. In many instances examples are given to show that the result fails for the next largest variety in the sequence.

Most of the main representation results have already been mentioned, but to get to them we must pass through many other results, some of independent interest. We shall mention a few of them here as part of a description of the contents of the paper.

In $\S 2$ we present the definitions of $\mathfrak{R e} U, \mathfrak{S b} U$, the four varieties of algebras mentioned above, representability and related concepts, and the class of representable relation algebras.

Sections 3 and 4 contain all the arithmetic needed for later results. For example, in $\S 3$ we show that the first identity below holds in every nonassociative relation algebra, while the remaining four hold in every semiassociative relation 
algebra:

$$
\begin{aligned}
1 & =1 ; 1, \\
(x ; y) ;(z ; 1) & =x ;(y ;(z ; 1)), \\
((1 ; x) ; y) ; z & =(1 ; x) ;(y ; z), \\
(x ;(y ; 1)) ; z & =x ;((y ; 1) ; z), \\
(x ;(1 ; y)) ; z & =x ;((1 ; y) ; z) .
\end{aligned}
$$

The last four equations are special cases of a much more general theorem, which states that if $\mathfrak{A}$ is a semiassociative relation algebra, then any rearrangement of parentheses is possible in a term of the form $x_{0} ; \ldots ; x_{\alpha-1}$, assuming that one of the $x_{\kappa}$ 's is 1 . For example, $(((w ; x) ; y) ; 1) ; z=w ;(x ;(y ;(1 ; z)))$. Thus, even though a semiassociative relation algebra $\mathfrak{A}$ may not satisfy the associative law, many of the consequences of that law will hold in $\mathfrak{A}$. It turns out that the proof of this associativity theorem for semiassociative relation algebras can be based entirely on the fact that every semiassociative relation algebra satisfies the five identities above. The associativity theorem is therefore obtained in $\S 4$ as a corollary of a similar result concerning a special class of groupoids with a distinguished element 1 , whose axioms are exactly the five identities above. The resulting associativity theorem for these special groupoids can be applied to cylindric algebras as well.

In $\S 5$ we show how the fundamental theorems about ideal elements, relativizations, and homomorphisms that are true for relation algebras carry over to semiassociative relation algebras, but not to weakly associative relation algebras. In particular, simplicity is characterized by a universal sentence for semiassociative relation algebras just as it is for relation algebras, so subalgebras of simple semiassociative relation algebras are also simple.

Section 6 contains two important results about representability and complete representability. First, we show that for relation algebras which have "finite products" the two concepts coincide. Second, we derive a necessary and sufficient condition for representability, namely that the algebra be embeddable in an atomic nonassociative relation algebra which has an $\omega$-dimensional relational basis. Relational bases and the basic facts about them are reviewed in that section. To get the second theorem we use Monk's results in [Mo70] on the existence of completions of relation algebras.

In $\S 7$ we define points, pairs, twins, functional elements, and identity atoms, and prove many things about them. That section contains generalizations of some theorems in [J82] and [SS85]. There is only one theorem in that section which holds for relation algebras but not for semiassociative relation algebras. This is exactly where to look to see why the algebra in (A) must be a relation algebra.

In $\S 8$ we study point-density and pair-density. Highlights from this section: every point-dense semiassociative relation algebra is a relation algebra; every simple pair-dense relation algebra is atomic; every simple pair-dense relation algebra has an $\omega$-dimensional relational basis. 
The concluding $\S 9$ contains all the representation results discussed above. One of the corollaries obtained there is

(H) $\mathfrak{A} \cong \mathfrak{R e}$ Pt $\mathfrak{A}$ iff $\mathfrak{A}$ is a simple complete point-dense semiassociative relation algebra.

This result generalizes Theorem 11 of [SS85] from relation algebras to semiassociative relation algebras. It also shows that one of the hypotheses of Theorem 11 is redundant. It is not necessary to assume that $\mathfrak{A}$ is atomic. The authors of [SS85] state that their Theorem 11 "simplifies the proof of a similar result given in a somewhat different environment by Maddux and Tarski". (The "similar result" is theorem (E) above.) Although Theorem 11 of [SS85] can be easily derived from theorem $(E)$, in a way similar to the proof of $(C)$ from $(E)$, it is not possible to go the other way around. Theorem (E) applies in a strictly larger class of algebras than Theorem 11 of [SS85]. It yields the representability of complex algebras of groups, but Theorem 11 does not. Thus it is not clear what the authors of [SS85] had in mind.

\section{Preliminaries}

In this section we define the classes of algebras with which we are concerned, and review some of their theory.

Definition 1. Let $\mathfrak{A}=\left\langle A,+, \cdot,^{-}, 0,1, ;,^{\smile}, 1^{\prime}\right\rangle$ be an algebra with binary operations,$+ \cdot$, and ; , unary operations ${ }^{-}$and ${ }^{-}$, and distinguished elements 0,1 , and 1 '. Then $\mathfrak{A}$ is a nonassociative relative algebra if $\langle A,+, \cdot,-, 0,1\rangle$ is a Boolean algebra (called the Boolean part of $\mathfrak{A}$ ), $\mathfrak{A}$ satisfies the identity law

(IL) $x=x ; 1^{\prime}=1^{\prime} ; x$,

and $\mathfrak{A}$ satisfies the cycle law

(CL) $x \cdot y ; z=0$ iff $y \cdot x ; \breve{z}=0$ iff $z \cdot \breve{y} ; x=0$.

NA is the class of all nonassociative relation algebras. Let $\mathfrak{A} \in$ NA. Then $\mathfrak{A}$ is a weakly associative relation algebra if it satisfies the weak associative law

(WL) $\left(\left(x \cdot 1^{\prime}\right) ; 1\right) ; 1=\left(x \cdot 1^{\prime}\right) ; 1$.

$\mathfrak{A}$ is a semiassociative relation algebra if it satisfies the semiassociative law

(SL) $(x ; 1) ; 1=x ; 1$.

$\mathfrak{A}$ is a relation algebra if it satisfies the associative law

(AL) $(x ; y) ; z=x ;(y ; z)$.

WA, SA, and RA are the classes consisting of all weakly associative relation algebras, all semiassociative relation algebras, and all relation algebras, respectively. If $\mathfrak{A} \in \mathrm{NA}$, then $0^{\prime}=1^{\prime-}$. The elements $1^{\prime}$ and $0^{\prime}$ are called the identity element and diversity element of $\mathfrak{A}$, respectively.

We will use "NA", "WA", "SA", and "RA" as abbreviations for the phrases "nonassociative relation algebra", "weakly associative relation algebra", "semiassociative relation algebra", and "relation algebra", respectively. 
Parentheses will be omitted from expressions involving operations in a NA according to the convention that the operations should be performed in the following order: ${ }^{-},-, ;, \cdot,+$. When the same binary operation occurs several times, the calculation should proceed from left to right. Thus, for example, $x ; y ; z=(x ; y) ; z$.

The cycle law is a more symmetrical way of stating De Morgan's Theorem K [D1864], mentioned in the Introduction. (Replace $z$ by $\bar{z}$, rearrange variables, and convert equalities to inequalities using Boolean algebra.) It was shown in [CT51] that relation algebras can be axiomatized by the IL, CL, and AL. This axiomatization was used in [JT52].

Note that the WL is a special case of the SL, so we obviously have NA $\supseteq$ WA $\supseteq$ SA. It turns out that $1=1 ; 1$ in any NA, so that the WL and the SL are also special cases of the AL. Furthermore, although these classes are not defined using equations (since the cycle law has the form of equivalences), they happen to be definable using equations. An equational definition for RA appears in [CT51], and equational definitions for NA, WA , and SA can be obtained from that one by eliminating the associative law, or replacing it with WL or SL.

Theorem 2. (i) NA, WA, SA, and RA are finitely based equational classes.

(ii) NA $\supsetneq W A \supsetneq S A \supsetneq R A$.

Proof. For part (i) see Theorem 1.5 of [Ma82]. For part (ii) see Theorems 1.3, $1.14,2.6$, and 3.7 of [Ma82].

Definition 3. For any equivalence relation $E$, let

$$
\mathfrak{S b} E=\left\langle\mathrm{Sb} E, \cup, \cap,{ }^{\sim}, \varnothing, E, \mid,{ }^{-1}, \operatorname{Id}_{\mathrm{Fd} E}\right\rangle,
$$

where, for all $R, S \subseteq E$,

$$
\begin{aligned}
\mathrm{Sb} E & =\{R: R \subseteq E\}, \\
\mathrm{Fd} E & =\{x: \text { for some } y,\langle x, y\rangle \in E \text { or }\langle y, x\rangle \in E\}, \\
\operatorname{Id}_{\mathrm{Fd} E} & =\{\langle x, x\rangle: x \in \mathrm{Fd} E\}, \\
R^{\sim E} & =E \sim R, \\
R \mid S & =\{\langle x, z\rangle: \text { for some } y,\langle x, y\rangle \in R \text { nd }\langle y, z\rangle \in S\}, \\
R^{-1} & =\{\langle y, x\rangle:\langle x, y\rangle \in R\} .
\end{aligned}
$$

Note that $\mathrm{Sb} E$ is closed under $\cup, \cap, \sim E,{ }^{-1}$, and $\mid . \mathfrak{S b} E$ is the (relation) algebra of subrelations of $E$. For any set $U$, let

$$
\mathfrak{R e} U=\mathfrak{S} \mathfrak{b}(U \times U) .
$$

$\mathfrak{R e} U$ is the (relation) algebra of relations on $U$.

Definition 4. Let $\mathfrak{A} \in \mathrm{NA}$ and let $E$ be an equivalence relation $\mathrm{A}$ function $R$ mapping $A$ into $\mathrm{Sb} E$ is a representation of $\mathfrak{A}$ over $E$ if $R$ is an isomorphic embedding of $\mathfrak{A}$ into $\mathfrak{S b} E$. In case $E=U \times U$ for some set $U$, we also 
say $R$ is a representation of $\mathfrak{A}$ over $U$. The representation $R$ is complete if it preserves all (possibly infinite) joins (and hence all meets). $\mathfrak{A}$ is representable if there is a representation of $\mathfrak{A}$ over some equivalence relation $E$, and $\mathfrak{A}$ is completely representable if there is a complete representation of $\mathfrak{A}$ over some equivalence relation. RRA is the class of all representable NA's.

There are representable relation algebras which are not completely representable. Thus they have representations, but no complete representations. (There are examples of such algebras in [Ma78a, L50].) For many algebras, however, the notions of representability and complete representability coincide. This is true for all finite algebras, and, as shown in $\S 6$, it is also true for any atomic algebra which has "finite products," i.e., the number of atoms below $x ; y$ is finite whenever the number of atoms below $x+y$ is finite.

Theorem 5. (i) RRA $\subsetneq$ RA,

(ii) RRA is the smallest equational class containing $\{\mathfrak{R e} U: U$ is a set $\}$,

(iii) RRA is not finitely axiomatizable,

(iv) RRA is recursively axiomatizable.

Proof. It is easy to check that RRA $\subseteq$ RA. The fact that the inclusion is proper was first proved by Lyndon [L50]. Part (ii) is due to Tarski [T55]. Part (iii) was proved by Monk [Mo64], using the connection between relation algebras and projective geometries discovered by Lyndon [L61]. A recursive axiomatization of RRA was constructed by Lyndon [L56].

\section{ELEMENTARY ARITHMETIC}

In this section and the next we develop the elementary arithmetic of NA's, WA's, and SA's to the extent necessary for later results in this paper. For each of these three classes this section contains theorems collecting various identities and implications for arbitrary elements and for atoms. The most extensive source of arithmetical results for relation algebras is [CT51]. Other good sources are [J82, JT52, Ma82].

The notions of complete additivity and normality for operators on Boolean algebras are defined in [JT51, Definition 1.1]. The next theorem states that ; and $\smile$ are completely additive and normal in every NA.

Theorem 6. Let $\mathfrak{A} \in \mathrm{NA}$.

(i) For all $X, Y \subseteq A$, if $\sum X$ and $\sum Y$ exist, then $\sum\{x ; y: x \in X, y \in Y\}$ also exists, and $\sum X ; \sum Y=\sum\{x ; y: x \in X, y \in Y\}$.

(ii) $x ; 0=0=0 ; x$ for every $x \in A$.

(iii) For every $X \subseteq A$, if $\sum X$ exists, then $\sum\{\breve{x}: x \in X\}$ also exists and $\left(\sum Y\right)^{\smile}=\sum\{\breve{x}: x \in X\}$.

(iv) $\breve{0}=0$.

Proof. Parts (i) and (ii) follow from Theorem 1.10 of [Ma82]. Parts (iii) and 
(iv) follow from Theorem 1.11 of [Ma82]. All four parts also follow from Theorems 2.3, 2.4, 2.4", 1.11 , and 1.6 of [CT51] (whose proofs do not use associativity).

The next definition gives relation algebraic analogues of the domain and range of a binary relation [Ma82, 5.10].

Definition 7. Let $\mathfrak{A} \in \mathrm{NA}$. For any $x \in A$ let $x^{\mathrm{d}}=1^{\prime} \cdot x ; \breve{x}$ and $x^{r}=1^{\prime} \cdot \breve{x} ; x$.

Now we turn to elementary laws for NA's that do not involve arbitrary joins or atoms. Parts (i)-(xvi), (xix)-(xx), and (xxvii)-(xxix) of the following theorem were first proved for relation algebras in [CT51].

The proofs in [CT51] for parts (i)-(xv) and (xxviii) do not use associativity, and can serve as proofs for the corresponding parts below. In addition, parts (i)-(xvi) are proved without associativity in [Ma82].

There is an essential but unmentioned use of associativity in Theorem 2.8 of [CT51], which states that the identity $a ; b \cdot c \leq a ; \breve{a} ; c$ holds in every RA. This identity happens to hold in every SA, but fails in some NA. However, the identity $a ; b \cdot c \leq a ;(\widetilde{a} ; c)$ holds in every NA. This alternative form (which follows from 1.22 and 2.7 , the theorems quoted in the proof of 2.8), together with the second law of duality (Theorem 1.24 of [CT51]) can be used to prove 2.9 of [CT51]. Theorem 2.10 (which coincides with part (xix) below) is proved in [CT51] by an appeal to 2.9, and therefore has a proof not using associativity. The same is true for Theorem 3.3, which also uses 2.9, and for part (xx), which is mentioned after Theorem 2.10 in [CT51].

Part (xvi) coincides with Theorem 3.12 of [CT51] (and with 1.13(15) in [Ma82]). The proof of 3.12 in [CT51] uses 3.11(i). The proof of 3.11(i) makes essential use of associativity by an appeal to 2.8 . The alternative form of 2.8 can be used instead, but certain other changes need to be made; see the proof of 1.13(15) in [Ma82].

The three conclusions of part (xxix) follow from Theorems 3.27, 3.26, and 4.1 of [CT51], respectively. The proof of 3.27 uses associativity via 2.9 , but the use of associativity in the proof of 2.9 is easily avoided, as was explained earlier. The proof of 3.26 uses 3.24 , and this latter theorem fails in some NA. However, associativity is required only to show that 3.24(ii) implies 3.24(i). The equivalence of 3.24(i) and 3.24(iii) is all that is needed in the proof of 3.26 , so the proof of 3.26 in [CT51] does not really use associativity. The third conclusion of part (xxix) follows from the equivalence of 4.1 (i) and 4.1(iii). The proof in [CT51] that 4.1(i) implies 4.1(iii) uses only the equivalence of 3.24(i) and 3.24(iii), and hence does not use associativity. The fact that 4.1(iii) implies 4.1(i) is not stated in (xxix), but it is used later in this paper. The proof that 4.1(iii) implies 4.1(i) in [CT51] has two unmentioned uses of associativity, in lines 12 and 16 , page 368 . Both of these uses can be easily eliminated by slight modifications. 
Theorem 8. Let $\mathfrak{A} \in \mathrm{NA}$. Then for all $v, w, x, y, z \in A$ :

(i) $(x+y) ; z=x ; z+y ; z$,

(ii) $x ;(y+z)=x ; y+x ; z$,

(iii) if $w \leq x$ and $y \leq z$, then $w ; y \leq x ; z$,

(iv) $x \leq x ; 1, x \leq 1 ; x$, and $x \leq 1 ; x ; 1$,

(v) $1=1 ; 1$,

(vi) $x ; y \cdot z \leq x ;(y \cdot \breve{x} ; z)$,

(vii) $x ; y \cdot z \leq(x \cdot z ; \breve{y}) ; y$,

(viii) $(x+y)^{\smile}=\breve{x}+\breve{y}$,

(ix) $x \leq y$ iff $\breve{x} \leq \breve{y}$,

(x) $(x \cdot \underline{y})^{\smile}=\breve{x} \cdot \breve{y}$,

(xi) $\bar{x}=\bar{x}$,

(xii) $0^{\smile}=0,1^{\smile}=1,1^{, \smile}=1^{\prime}$, and $0^{\prime} \smile=0$ ',

(xiii) if $x \leq 1$, then $\breve{x}=x$,

(xiv) $x^{\smile}=x$,

(xv) $(x ; y)^{\smile}=\breve{y} ; \breve{x}$,

(xvi) if $x, y \leq 1$ ' then $x \cdot y=x ; y$,

(xvii) if $x \leq 1$, then $x ;(y \cdot z)=x ; y \cdot z=y \cdot z \cdot x ; 1$ and $(y \cdot z) ; x=$ $y ; x \cdot z=y \cdot z \cdot 1 ; x$,

(xviii) if $x, y \leq 1$ ' then $x ; 1 ; y=x ; 0 ; y+x \cdot y$,

(xix) $x \cdot y \cdot z \leq x ; \breve{y} ; z$,

(xx) $v \cdot w \cdot x \cdot y \cdot z \leq v ; \breve{w} ; x ; \breve{y} ; z$,

(xxi) $x^{\mathrm{d}}=1^{\prime} \cdot x ; \breve{x}=1^{\prime} \cdot x ; 1=1^{\prime} \cdot 1 ; \breve{x}$,

(xxii) $x^{r}=1^{\prime} \cdot \breve{x} ; x=1^{\prime} \cdot 1 ; x=1, \cdot \breve{x} ; 1$,

(xxiii) $(\breve{x})^{\mathrm{d}}=x^{\mathrm{r}}$ and $(\breve{x})^{\mathrm{r}}=x^{\mathrm{d}}$,

(xxiv) $x=x^{\mathrm{d}} ; x=x ; x^{r}$,

(xxv) if $x \leq 1$ ' then $x=x^{\mathrm{d}}=x^{\mathrm{r}}$,

(xxvi) if $x ; 1 ; x \leq 1$, then $x \leq 1, x ; 0 ; x=0, x ; 1 ; x=x$,

(xxvii) if $x ; 0^{\prime} ; x ; 0^{\prime} ; x \leq 1$ ' then $x \leq 1$ ',

(xxviii) $\breve{x} ; \bar{x} \leq 0^{\prime}, \bar{x} ; \breve{x} \leq 0$ ', $x ; \overline{\bar{x}} \leq 0^{\prime}$, and $\overline{\bar{x}} ; x \leq 0^{\prime}$,

(xxix) if $x=x ; 1=1 ; x$, then $\bar{x}=x, \bar{x}=\bar{x} ; 1=1 ; \bar{x}$, and $x \cdot y ; x=$ $(x \cdot y) ;(x \cdot z)$.

Proof. Parts (i)-(xvi) and (xxiii)-(xxv) are proved in Theorems 1.4, 1.13, and 5.11 of [Ma82].

(xvii): Assume $x \leq 1$ '. The proof of the first group of identities goes as follows. 


$$
\begin{aligned}
x ;(y \cdot z) & \leq 1^{\prime} ; y \cdot 1^{\prime} ; z \cdot x ; 1 & & \left(\text { iii) }, x \leq 1^{\prime}\right. \\
& =y \cdot z \cdot x ; 1 & & \text { IL } \\
& \leq z \cdot x ;(1 \cdot \breve{x} ; y) & & (\mathrm{vi}) \\
& \leq z \cdot x ;\left(1^{,-} ; y\right) & & (\text { iii }),(\mathrm{ix}), x \leq 1 \\
& =z \cdot x ; y & & (\mathrm{xii}), \mathrm{IL} \\
& \leq x ;(y \cdot \breve{x} ; z) & & \text { (vi) } \\
& =x ;(y \cdot z) & & \text { (iii), (ix), (xii), IL, } x \leq 1 .
\end{aligned}
$$

The second group of identities can be easily derived from the first group by using parts (x), (xiii) $-(\mathrm{xv})$.

(xviii): Assume $x, y \leq 1^{\prime}$. Then

$$
\begin{aligned}
x ; 1 ; y & =x ; 0^{\prime} ; y+x ; 1^{\prime} ; y & & (\mathrm{i}),(\mathrm{ii}) \\
& =x ; 0^{\prime} ; y+x \cdot y & & \text { IL, (xvi) }
\end{aligned}
$$

(xix):

$$
\begin{aligned}
x \cdot y \cdot z & =(x \cdot y \cdot z) ; 1^{\prime} & & \text { IL } \\
& \leq(x \cdot y \cdot z) ;\left(1^{\prime} \cdot(x \cdot y \cdot z)^{\smile} ;(x \cdot y \cdot z)\right) & & \text { (vi) } \\
& \leq x ; \breve{y} ; z & & \text { (iii), (ix) } .
\end{aligned}
$$

(xx): Use parts (iii) and (xix) twice.

(xxi):

$$
\begin{aligned}
x^{\mathrm{d}} & =1^{\prime} \cdot x ; \breve{x} \leq 1^{\prime} \cdot x ; 1 & & (\mathrm{iii}) \\
& \leq 1^{\prime} \cdot x ;\left(1 \cdot \breve{x} ; 1^{\prime}\right) & & (\mathrm{vi}) \\
& =1^{\prime} \cdot x ; \breve{x} & & (\mathrm{IL}) \\
& \leq 1^{\prime} \cdot 1 ; \breve{x} & & (\mathrm{iii}) \\
& \leq 1^{\prime} \cdot\left(1 \cdot 1^{\prime} ; x^{\smile}\right) ; \breve{x} & & (\mathrm{v}) \\
& =1^{\prime} \cdot x ; \breve{x} & & \text { IL, (xiv). }
\end{aligned}
$$

(xxii): The proof is similar to that of part (xxi).

(xxvi): If $x ; 0 ; x \leq 1$ ', then

$$
\begin{array}{rlrl}
x \cdot 0^{\prime} & \leq\left(x \cdot 0^{\prime}\right) ;\left(x \cdot 0^{\prime}\right)^{\smile} ;\left(x \cdot 0^{\prime}\right) & & (\mathrm{xix}) \\
& \leq x ; 0^{\prime} ; x & & \text { (iii), (ix), (xii) } \\
& \leq 1^{\prime}, &
\end{array}
$$

so $x \leq 1^{\prime}$. Hence $x ; 0^{\prime} ; x \leq 1^{\prime} \cdot 1^{\prime} ; 0^{\prime} ; 1^{\prime}=1^{\prime} \cdot 0^{\prime}=0$ by part (iii) and IL. Finally, by part (xviii), $x ; 1 ; x=x ; 0 ; x+x=0+x=x$. 
(xxvii): The proof is similar to the first part of the proof of (xxvi), but uses part $(\mathbf{x x})$ in place of (xix).

(xxviii): By the identity law, $0=x \cdot \bar{x}=x ; 1^{\prime} \cdot \bar{x}$, so $0=\bar{x} ; \bar{x} \cdot 1^{\prime}$ by the cycle law. Thus $\bar{x} ; \bar{x} \leq 0$ '. The other inequalities can be obtained similarly.

(xxix): Assume $x=x ; 1=1 ; x$. Then

$$
\begin{aligned}
\breve{x} & \leq \breve{x} ; x ; \breve{x} & & (\mathrm{xix}),(\mathrm{xiv}) \\
& \leq 1 ; x ; 1=x ; 1=x & & \text { (iii) },
\end{aligned}
$$

so $\breve{x}=x$ by parts (ix) and (xiv). Note that $0=x \cdot \bar{x}=x ; 1 \cdot \bar{x}$, so $0=\bar{x} ; 1 \cdot x$ by the cycle law and part (xii). Thus $\bar{x} ; 1 \leq \bar{x}$, and, similarly, $1 ; \bar{x} \leq \bar{x}$. The opposite inclusions hold by part (iv), so $\bar{x}=\bar{x} ; 1=1 ; \bar{x}$. Finally,

$$
\begin{aligned}
x \cdot y ; z & \leq x \cdot y ;(z \cdot \breve{y} ; x) \\
& \leq x \cdot y ;(z \cdot 1 ; x)=x \cdot y ;(z \cdot x) \\
& \leq\left(y \cdot x ;(z \cdot x)^{\smile}\right) ;(x \cdot z) \\
& \leq(y \cdot x ; 1) ;(x \cdot z)=(x \cdot y) ;(x \cdot z) \\
& \leq x ; 1 \cdot y ; z \\
& =x \cdot y ; z .
\end{aligned}
$$

If any identity holds in a nonassociative relation algebra $\mathfrak{A}$, then so does the dual of that identity. The dual of an identity is obtained by replacing every subterm of the form $x ; y$ by $y ; x$. For example, the dual of the SL is $1 ;(1 ; x)=1 ; x$. The dual of an identity can be proved by using that identity together with parts (xiv) and (xv). (This is the "second dual" of [CT51, p. 349].) The proof of the dual of the SL is $1 ;(1 ; x)=1^{\smile} ;\left(1^{\smile} ; x^{\smile}\right)=$ $\left(\breve{x} ; 1^{\smile} ; 1^{\smile}\right)^{\smile}=\left(\breve{x} ; 1^{\smile}\right)^{\smile}=1^{\smile} ; x^{\smile}=1 ; x$. (See Theorem 1.24 of [CT51], the "second law of duality".)

Definition 9. Let $\mathfrak{A} \in N A$. Then At $\mathfrak{A}$ is the set of atoms (of the Boolean part) of $\mathfrak{A}$.

Theorem 10. Let $\mathfrak{A} \in \mathrm{NA}$.

(i) If $x \in$ At $\mathfrak{A}$ then $\breve{x} \in$ At $\mathfrak{A}$.

(ii) If $x, y, z \in$ At $\mathfrak{A}$ then $x \leq y$; $z$ iff $y \leq x ; \breve{z}$ iff $z \leq \breve{y} ; x$.

Proof. Part (i) is Theorem 3.4 of [Ma82], and also Theorem 4.3(xxi) of [JT52], whose proof depends on proofs in [CT51] which do not use associativity. Part (ii) is an immediate consequence of the cycle law.

Theorems 11-12 below contain results which hold in all relation algebras but which do not appear in either [CT51] or [JT52]. Their proofs require associativity, since they do not hold in every NA. The weak associative law suffices for their proofs, so they hold in every WA. Theorem 11 is used to prove Theorem 12 , and Theorem 12 will be used much later in this paper. 
Theorem 11. Let $\mathfrak{A} \in W A$. Then for all $x, y \in A$ :

(i) $x^{\mathrm{d}} ; 1=x ; 1 ; 1=x ; 1 ; 1 ; 1$ and $1 ; x^{\mathrm{r}}=1 ;(1 ; x)=1 ;(1 ;(1 ; x))$,

(ii) $x^{\mathrm{d}}=(x ; 1)^{\mathrm{d}}$ and $x^{r}=(1 ; x)^{r}$,

(iii) if $x \leq y$; 1 then $x^{\mathrm{d}} \leq y^{\mathrm{d}}$, and if $x \leq 1 ; y$, then $x^{r} \leq y^{r}$.

Proof. By duality, we need only prove the first half of each part.

$$
\begin{aligned}
x^{\mathrm{d}} ; 1 & =(1 \cdot x ; \breve{x}) ; 1 & & \\
& \leq x ; 1 ; 1 & & \text { Theorem 8(iii) } \\
& \leq x ; 1 ; 1 ; 1 & & \text { Theorem 8(iv) } \\
& =x^{\mathrm{d}} ; x ; 1 ; 1 ; 1 & & \text { Theorem 8(xxiv) } \\
& \leq x^{\mathrm{d}} ; 1 ; 1 ; 1 ; 1 & & \text { Theorem 8(iii) } \\
& \leq x^{\mathrm{d}} ; 1 ; 1 ; 1 & & \text { WL } \\
& \leq x^{\mathrm{d}} ; 1 ; 1 & & \text { WL } \\
& \leq x^{\mathrm{d}} ; 1 & & \text { WL. }
\end{aligned}
$$

(ii):

$$
\begin{aligned}
(x ; 1)^{\mathrm{d}} & =1^{\prime} \cdot x ; 1 ; 1 & & \text { Theorem } 8(\mathrm{xxi}) \\
& =1^{\prime} \cdot x^{\mathrm{d}} ; 1 & & (\mathrm{i}) \\
& =x^{\mathrm{dd}} & & \text { Theorem } 8(\mathrm{xxi}) \\
& =x^{\mathrm{d}} & & \text { Theorem } 8(\mathbf{x x v}) .
\end{aligned}
$$

(iii): Assume $x \leq y ; 1$. Then, by part (ii) and Theorem 8(iii)(ix), we have $x^{\mathrm{d}} \leq(y ; 1)^{\mathrm{d}}=y^{\mathrm{d}}$.

Richard L. Kramer discovered that the identity $x ; 1 ; 1=x ; 1 ; 1 ; 1$ and its dual hold in every WA.

Theorem 12. Let $\mathfrak{A} \in \mathrm{WA}$. Then for all $x, y \in$ At $\mathfrak{A}$ :

(i) $x^{\mathrm{d}}, x^{\mathrm{r}} \in$ At $\mathfrak{A}$,

(ii) $x^{\mathrm{d}}=y$ iff $y \leq 1$, and $y ; x=x$.

(iii) $x^{r}=y$ iff $y \leq 1$, and $x ; y=x$,

(iv) if $x ; y \neq 0$ then $x^{r}=y^{\mathrm{d}}$,

(v) if $x \leq y$; 1 then $x^{\mathrm{d}}=y^{\mathrm{d}}$,

(vi) if $x \leq 1 ; y$ then $x^{r}=y^{r}$.

Proof. For parts (i)-(iii), see [Ma82, Theorem 5.12]. The proofs of parts (iv)(vi) are very similar. We only prove (v). Suppose $x \leq y ; 1$. By Theorem 8(iii)(ix) and Theorem 11(ii), $x^{\mathrm{d}} \leq(y ; 1)^{\mathrm{d}}=y^{\mathrm{d}}$, by $x^{\mathrm{d}}, y^{\mathrm{d}} \in$ At $\mathfrak{A}$ by part (i), so $x^{\mathrm{d}}=y^{\mathrm{d}}$.

The identities in Theorem 13 require the semiassociative law, since they do not hold in every WA. Part (i) does not appear in [CT51] or [JT52], but does 
appear in [TG87, 3.2(xvi)]. Part (ii) follows from the semiassociative law and the proof of Theorem 5.2 of [CT51]. Parts (iv)-(vi) are trivial consequences of full associativity.

Theorem 13. Let $\mathfrak{A} \in \mathrm{SA}$. Then for all $x, y, z \in A$ :

(i) $(x \cdot y ; z) ; 1=(y \cdot x ; \breve{z}) ; 1$ and $1 ;(x \cdot y ; z)=1 ;(z \cdot \breve{y} ; x)$,

(ii) $x ; y \cdot 1 ; z=x ;(y \cdot 1 ; z)$ and $x ; y \cdot z ; 1=(x \cdot z ; 1) ; y$,

(iii) $(x \cdot 1 ; \breve{y}) ; z=x ;(z \cdot y ; 1)$,

(iv) $x ; y ; 1=x ;(y ; 1)$ and $1 ; x ; y=1 ;(x ; y)$,

(v) $x ; y ;(z ; 1)=x ;(y ;(z ; 1))$ and $1 ; x ; y ; z=1 ; x ;(y ; z)$,

(vi) $x ;(y ; 1) ; z=x ;(y ; 1 ; z)$ and $x ;(1 ; y) ; z=x ;(1 ; y ; z)$,

(vii) $1 ;(1 ; x ; 1) ; 1=1 ; x ; 1$,

(viii) $1 ;(1 ; x ; 1)^{-} ; 1=(1 ; x ; 1)^{-}$.

Proof. To prove (i), (ii), (iv), (v), and (vi) we need only prove the first identity in each case, since the second identity follows from the first by duality.

(i):

$$
\begin{aligned}
(x \cdot y ; z) ; 1 & \leq(y \cdot x ; \breve{z}) ; z ; 1 & & \text { Theorem 8(iii)(vii) } \\
& \leq(y \cdot x ; \breve{z}) ; 1 ; 1 & & \text { Theorem 8(iii) } \\
& \leq(y \cdot x ; \breve{z}) ; 1 & & \text { SL } \\
& \leq(x \cdot y ; z-\smile) ; \breve{z} ; 1 & & \text { Theorem 8(iii)(vii) } \\
& \leq(x \cdot y ; z) ; 1 ; 1 & & \text { Theorem 8(iii)(xiv) } \\
& \leq(x \cdot y ; z) ; 1 & & \text { SL. }
\end{aligned}
$$

(ii):

$$
\begin{aligned}
x ; y \cdot 1 ; z & =x ;(y \cdot \breve{x} ;(1 ; z)) & & \text { Theorem } 8(\mathrm{vi}) \\
& \leq x ;(y \cdot 1 ;(1 ; z)) & & \text { Theorem } 8(\mathrm{iii}) \\
& =x ;(y \cdot 1 ; z) & & \text { dual of SL } \\
& \leq x ; y \cdot 1 ;(1 ; z) & & \text { Theorem } 8(\mathrm{iii}) \\
& \leq x ; y \cdot 1 ; z & & \text { dual of SL. }
\end{aligned}
$$

(iii):

$$
\begin{aligned}
(x \cdot 1 ; \breve{y}) ; z & \leq(x \cdot 1 ; \breve{y}) ;\left(z \cdot(x \cdot 1 ; \breve{y})^{\smile} ; 1\right) & & \text { Theorem } 8(\mathrm{vi}) \\
& \leq x ;(z \cdot y ; 1 ; 1) & & \text { Theorem } 8(\mathrm{iii})(\mathrm{ix})(\mathrm{xii})(\mathrm{xiv})(\mathrm{xv}) \\
& \leq x ;(z \cdot y ; 1) & & \text { SL } \\
& \leq\left(x \cdot 1 ;(z \cdot y ; 1)^{\smile}\right) ;(z \cdot y ; 1) & & \text { Theorem } 8(\text { vii }) \\
& \leq(x \cdot 1 ;(1 ; \breve{y})) ; z & & \text { Theorem } 8(\mathrm{iii})(\mathrm{ix})(\mathrm{xii})(\mathrm{xv}) \\
& \leq(x \cdot 1 ; \breve{y}) ; z & & \text { dual of SL. }
\end{aligned}
$$


(iv):

$$
\begin{aligned}
x ; y ; 1 & =(1 \cdot x ; y) ; 1 \\
& =(x \cdot 1 ; \breve{y}) ; 1 \\
& =x ;(1 \cdot y ; 1) \\
& =x ;(y ; 1) .
\end{aligned}
$$

(v):

$$
\begin{aligned}
x ; y ;(z ; 1) & =x ; y ; z ; 1 \\
& =(1 \cdot x ; y ; z) ; 1 \\
& =(x ; y \cdot 1 ; \breve{z}) ; 1 \\
& =x ;(y \cdot 1 ; \breve{z}) ; 1 \\
& =x ;((y \cdot 1 ; \breve{z}) ; 1) \\
& =x ;((1 \cdot y ; z) ; 1) \\
& =x ;(y ; z ; 1) \\
& =x ;(y ;(z ; 1))
\end{aligned}
$$

(vi):

$$
\begin{aligned}
x ;(y ; 1) ; z & =x ; y ; 1 ; z \\
& =x ; y ; 1 \cdot 1 ; z \\
& =x ;(y ; 1) \cdot 1 ; z \\
& =x ;(y ; 1 \cdot 1 ; z) \\
& =x ;(y ; 1 ; z)
\end{aligned}
$$

(vii): By part (iv) and Theorem $8(\mathrm{v})$.

(viii): By part (vii) and Theorem 8(iv)(xxix).

\section{AsSOCIATIVITY IN GROUPOIDS}

In this section we will prove a general associativity result for semiassociative relation algebras. This result is a consequence of just Theorems $8(v)$ and $13(\mathrm{v})(\mathrm{vi})$. Thus it has independent interest as a result above associativity in groupoids having a distinguished element. Of course, all uses of Theorem 25 later in this paper could be replaced by short derivations involving $8(\mathrm{v})$ and 13(iv)(v)(vi). (As shown below, Theorem 13(iv) follows from 8(v) and 13(v).)

Definition 14. A groupoid with distinguished element is an algebra of the form $\mathfrak{A}=\langle A, 1, ;\rangle$ where $1 \in A$ and ; is a binary operation on $A$. Let GD be the class of all groupoids with distinguished element.

Definition 15. For every $\mathfrak{A} \in \mathrm{GD}$, let $P^{(\mathfrak{A})}$ be the unique function which maps finite sequences of elements of $A$ to subsets of $A$, i.e., $P^{(\mathfrak{A})}:\left(\bigcup_{n=0}^{\omega}{ }^{n} A\right) \rightarrow$ $\mathrm{Sb}(A)$, and which satisfies the following conditions: 
$P(x)=\{x\}$ for every $x \in A$,

$P\left(x_{0}, \ldots, x_{n-1}\right)=\left\{y ; z: y \in P\left(x_{0}, \ldots, x_{m-1}\right), z \in P\left(x_{m}, \ldots, x_{n-1}\right)\right.$, $0<m<n\}$, whenever $x_{0}, \ldots, x_{n-1} \in A$.

The notation $P\left(x_{0}, \ldots, x_{n-1}\right)$ will be often used in place of the notation $P^{(\mathfrak{A})}\left(x_{0}, \ldots, x_{n-1}\right)$ if the algebra involved is not relevant or else clear from context. The simplest cases of the definition of $P$ are

$$
\begin{array}{r}
P(x)=\{x\}, \quad P(x, y)=\{x ; y\}, \quad P(x, y, z)=\{(x ; y) ; z, x ;(y ; z)\} \\
P(w, x, y, z)=\{w ; x ; y ; z, w ; x ;(y ; z), w ;(x ;(y ; z)) \\
w ;(x ; y ; z), w ;(x ; y) ; z\} .
\end{array}
$$

Definition 16. Let $\mathrm{GA}$ be the class of $\mathfrak{A}=\langle A, 1, ;\rangle \in \mathrm{GD}$ such that, for all $x, y, z \in A$ :
(A1) $1=1 ; 1$,
(A2) $1 ; x ; y ; z=1 ; x ;(y ; z)$,
(A3) $x ;(1 ; y) ; z=x ;(1 ; y ; z)$,
(A4) $x ;(y ; 1) ; z=x ;(y ; 1 ; z)$,
(A5) $x ; y ;(z ; 1)=x ;(y ;(z ; 1))$.

Algebras in GA are "groupoids with some associativity".

Lemma 17. Let $\mathfrak{A} \in \mathrm{GA}$. Then for all $x, y \in A$ :
(i) $1 ; x ; y=1 ;(x ; y)$,
(ii) $x ; 1 ; y=x ;(1 ; y)$,
(iii) $x ; y ; 1=x ;(y ; 1)$.

Proof. Part (i) follows from (A1) and (A2): $1 ; x ; y=1 ; 1 ; x ; y=1 ; 1 ;(x ; y)$ $=1 ;(x ; y)$. Similarly, part (ii) follows from (A1) and either (A3) or (A4), and part (iii) is a consequence of (A1) and (A5).

Lemma 18. Suppose $\mathfrak{A} \in \mathrm{GA}$, and $1 \in\{w, x, y, z\} \subseteq A$. Then $|P(w, x, y, z)|$ $=1$.

Proof. We must show that the five elements of $P(w, x, y, z)$ coincide in each of these four cases: $w=1, x=1, y=1$, and $z=1$.

$$
\begin{aligned}
1 ;(x ;(y ; z)) & =1 ; x ;(y ; z) & & \text { Lemma 17(i) } \\
& =1 ; x ; y ; z & & (\mathrm{~A} 2) \\
& =1 ;(x ; y) ; z & & \text { Lemma 17(i) } \\
& =1 ;(x ; y ; z) & & \text { Lemma 17(i). }
\end{aligned}
$$




$$
\begin{aligned}
w ; 1 ; y ; z & =w ;(1 ; y) ; z & & \text { Lemma 17(ii) } \\
& =w ;(1 ; y ; z) & & (\mathrm{A} 3) \\
& =w ;(1 ;(y ; z)) & & \text { Lemma 17(i) } \\
& =w ; 1 ;(y ; z) & & \text { Lemma 17(ii). } \\
w ;(x ;(1 ; z)) & =w ;(x ; 1 ; z) & & \text { Lemma 17(ii) } \\
& =w ;(x ; 1) ; z & & (\mathrm{~A} 4) \\
& =w ; x ; 1 ; z & & \text { Lemma 17(iii) } \\
& =w ; x ;(1 ; z) & & \text { Lemma 17(ii). } \\
w ; x ; y ; 1 & =w ; x ;(y ; 1) & & \text { Lemma 17(iii) } \\
& =w ;(x ;(y ; 1)) & & \text { (A5) } \\
& =w ;(x ; y ; 1) & & \text { Lemma 17(iii) } \\
& =w ;(x ; y) ; 1 & & \text { Lemma 17(iii). }
\end{aligned}
$$

Definition 19. For every $\mathfrak{A} \in \mathrm{GD}$, let $\Gamma(\mathfrak{A})=\{x ; 1 ; y: x, y \in A\}$.

Note that $\Gamma(\mathfrak{A})=\{x ;(1 ; y): x, y \in A\}$ by Lemma $17(\mathrm{ii})$.

Lemma 20. Let $\mathfrak{A} \in \mathrm{GA}$. Then

(i) $1 \in \Gamma(\mathfrak{A})$,

(ii) if $x \in \Gamma(\mathfrak{A})$ then $x ; y \in \Gamma(\mathfrak{A})$ and $y ; x \in \Gamma(\mathfrak{A})$ for every $y \in A$.

Proof. Part (i) follows immediately from (A1). For part (ii), assume $x \in \Gamma(\mathfrak{A})$. Then there are $v, w \in A$ such that $x=v ; 1 ; w$. For every $y \in A$, Lemma 18 implies

$$
\begin{aligned}
& x ; y=v ; 1 ; w ; y=v ; 1 ;(w ; y) \in \Gamma(\mathfrak{A}) \\
& y ; x=y ;(v ; 1 ; w)=(y ; v) ; 1 ; w \in \Gamma(\mathfrak{A})
\end{aligned}
$$

Lemma 21. Suppose $\mathfrak{A} \in \mathrm{GA}, x, y, z \in A$, and $\Gamma(\mathfrak{A}) \cap\{x, y, z\} \neq \varnothing$. Then $|P(x, y, z)|=1$, i.e., $x ; y ; z=x ;(y ; z)$.

Proof. By the assumptions, there are $v, w \in A$ such that $v ; 1 ; w \in\{x, y, z\}$. The following derivations for the resulting three cases use various instances of Lemma 18.

If $v ; 1 ; w=x$, then

$$
\begin{aligned}
x ; y ; z & =v ; 1 ; w ; y ; z & & \\
& =v ; 1 ;(w ; y) ; z & & |P(v, 1, w, y)|=1 \\
& =v ;(1 ;(w ; y) ; z) & & |P(v, 1, w ; y, z)|=1 \\
& =v ;(1 ; w ;(y ; z)) & & |P(1, w, y, z)|=1 \\
& =v ; 1 ; w ;(y ; z) & & |P(v, 1, w, y ; z)|=1 \\
& =x ;(y ; z) . & &
\end{aligned}
$$


If $v ; 1 ; w=y$, then

$$
\begin{aligned}
x ; y ; z & =x ;(v ; 1 ; w) ; z & & \\
& =x ; v ; 1 ; w ; z & & |P(x, v, 1, w)|=1 \\
& =x ; v ; 1 ;(w ; z) & & |P(x ; v, 1, w, z)|=1 \\
& =x ;(v ; 1 ;(w ; z)) & & |P(x, v, 1, w ; z)|=1 \\
& =x ;(v ; 1 ; w ; z) & & |P(v, 1, w, z)|=1 \\
& =x ;(y ; z) . & &
\end{aligned}
$$

Finally, if $v ; 1 ; w=z$, then

$$
\begin{aligned}
x ; y ; z & =x ; y ;(v ; 1 ; w) & & \\
& =x ; y ; v ; 1 ; w & & |P(x ; y, v, 1, w)|=1 \\
& =x ;(y ; v ; 1) ; w & & |P(x, y, v, 1)|=1 \\
& =x ;(y ; v ; 1 ; w) & & |P(x, y ; v, 1, w)|=1 \\
& =x ;(y ;(v ; 1 ; w)) & & |P(y, v, 1, w)|=1 \\
& =x ;(y ; z) . & &
\end{aligned}
$$

Lemma 22. Suppose $\mathfrak{A} \in \mathrm{GA}$ and $y \in \Gamma(\mathfrak{A})$. Then for all $u, v, w, x_{0}, \ldots$, $x_{n-1} \in A$,

$$
u ;(v ; w) ; x_{0} ; \ldots ; x_{n-1} ; y=u ; v ; w ; x_{0} ; \ldots ; x_{n-1} ; y .
$$

Proof. The proof is by induction on $n$. Suppose $n=0$, i.e., there are no $x_{k}$ 's. Note that $w ; y \in \Gamma(\mathfrak{A})$ by Lemma 20(ii). Hence,

$$
\begin{aligned}
& u ;(v ; w) ; y=u ;(v ; w ; y) \quad \text { Lemma } 21, y \in \Gamma(\mathfrak{A}) \\
& =u ;(v ;(w ; y)) \quad \text { Lemma } 21, y \in \Gamma(\mathfrak{A}) \\
& =u ; v ;(w ; y) \quad \text { Lemma } 21, w ; y \in \Gamma(\mathfrak{A}) \\
& =u ; v ; w ; y \quad \text { Lemma } 21, y \in \Gamma(\mathfrak{A}) \text {. }
\end{aligned}
$$

Now we assume the lemma holds for $n$, and show that it also holds for $n+1$. We have $x_{n} ; y \in \Gamma(\mathfrak{A})$ by Lemma 20(ii), so we get

$$
\begin{array}{rlrl}
u ;(v ; w) & ; x_{0} ; \ldots ; x_{n-1} ; x_{n} ; y & \\
& =u ;(v ; w) ; x_{0} ; \ldots ; x_{n-1} ;\left(x_{n} ; y\right) & & \text { Lemma } 21, y \in \Gamma(\mathfrak{A}) \\
& =u ; v ; w ; x_{0} ; \ldots ; x_{n-1} ;\left(x_{n} ; y\right) & & \text { inductive hypothesis } \\
& =u ; v ; w ; x_{0} ; \ldots ; x_{n-1} ; x_{n} ; y & & \text { Lemma } 21, y \in \Gamma(\mathfrak{A}) .
\end{array}
$$

Lemma 23. Suppose $\mathfrak{A} \in \mathrm{GA}$ and $x_{0}, \ldots, x_{n-1} \in A$. If $\Gamma(\mathfrak{A}) \cap\left\{x_{0}, \ldots, x_{n-1}\right\}$ $\neq \varnothing$, then $\left|P\left(x_{0}, \ldots, x_{n-1}\right)\right|=1$.

Proof. Use induction on $n$. If $n=1$ or $n=2$, then the conclusion obviously holds, even if the hypothesis does not. So we will assume the lemma holds for a given $n>2$, and show that it also holds for $n+1$. Notice that $P^{(\mathfrak{A})}$ has the following fundamental property. 


$$
\begin{aligned}
P\left(x_{0}, \ldots, x_{n}\right)= & \bigcup_{m=0}^{n-1} P\left(x_{0}, \ldots, x_{m-1}, x_{m} ; x_{m+1}, x_{m+2}, \ldots, x_{n}\right) \\
= & P\left(x_{0} ; x_{1}, x_{2}, \ldots, x_{n}\right) \cup P\left(x_{0}, x_{1} ; x_{2}, x_{3}, \ldots, x_{n}\right) \\
& \cup \cdots \cup P\left(x_{0}, \ldots, x_{n-2}, x_{n-1} ; x_{n}\right) .
\end{aligned}
$$

Since we are assuming the lemma holds for $n$, (1) yields

$$
\begin{aligned}
P\left(x_{0}, \ldots, x_{n}\right)=\{ & \left.x_{0} ; x_{1} ; x_{2} ; \ldots ; x_{n}\right\} \\
& \cup\left\{x_{0} ;\left(x_{1} ; x_{2}\right) ; x_{3} ; \ldots ; x_{n}\right\} \\
& \cup \cdots \cup\left\{x_{0} ; \ldots ; x_{n-2} ;\left(x_{n-1} ; x_{n}\right)\right\} .
\end{aligned}
$$

The proof will therefore be complete if we manage to show, whenever $0<m<$ $n$, that

$$
x_{0} ; x_{1} ; \ldots ; x_{n}=x_{0} ; \ldots ; x_{m-1} ;\left(x_{m} ; x_{m+1}\right) ; x_{m+2} ; \ldots ; x_{n} .
$$

By hypothesis, $\Gamma(\mathfrak{A}) \cap\left\{x_{0}, \ldots, x_{n}\right\} \neq \varnothing$, so assume $y \in \Gamma(\mathfrak{A})$ and $y \in$ $\left\{x_{0}, \ldots, x_{n}\right\}$. We now consider two cases.

If $y \in\left\{x_{0}, \ldots, x_{m+1}\right\}$, then, by Lemma 20(ii),

$$
\Gamma(\mathfrak{A}) \cap\left\{x_{0} ; \ldots ; x_{m-1}, x_{m}, x_{m+1}\right\} \neq \varnothing,
$$

so, by Lemma 21 ,

$$
x_{0} ; \ldots ; x_{m-1} ;\left(x_{m} ; x_{m+1}\right)=x_{0} ; \ldots ; x_{m-1} ; x_{m} ; x_{m+1},
$$

and (3) follows immediately from (4).

If $y \in\left\{x_{m+2}, \ldots, x_{n}\right\}$, say $y=x_{k} \in \Gamma(\mathfrak{A})$ with $m+2 \leq k \leq n$, then, by Lemma 22,

$$
\begin{aligned}
& x_{0} ; \ldots ; x_{m-1} ;\left(x_{m} ; x_{m+1}\right) ; x_{m+2} ; \ldots ; x_{k} \\
& \quad=x_{0} ; \ldots ; x_{m-1} ; x_{m} ; x_{m+1} ; x_{m+2} ; \ldots ; x_{k},
\end{aligned}
$$

and (5) implies (3).

Theorem 24. Assume $\mathfrak{A}=\langle A, 1, ;\rangle \in \mathrm{GA}, 0<n<\omega$, and $x_{0}, \ldots, x_{n-1} \in$ $A$. If $\Gamma(\mathfrak{A}) \cap\left\{x_{0}, \ldots, x_{n-1}\right\} \neq \varnothing$, in particular, if $1 \in\left\{x_{0}, \ldots, x_{n-1}\right\}$, then $\left|P^{(\mathfrak{A})}\left(x_{0}, \ldots, x_{n}\right)\right|=1$.

Proof. This theorem follows immediately from Lemma 20(i) and Lemma 23.

Theorem 25. If $\mathfrak{A}=\left\langle A,+, \cdot,^{-}, 0,1, ;,^{\smile}, 1^{\prime}\right\rangle \in \mathrm{SA}$, then $\langle A, 1, ;\rangle \in \mathrm{GA}$. If $0<n<\omega, x_{0}, \ldots, x_{n-1} \in A$, and $\Gamma(\langle A, 1, ;\rangle) \cap\left\{x_{0}, \ldots, x_{n-1}\right\} \neq \varnothing$, in particular, if $1 \in\left\{x_{0}, \ldots, x_{n-1}\right\}$, then $\left|P^{(\mathfrak{A})}\left(x_{0}, \ldots, x_{n-1}\right)\right|=1$.

Proof. By Theorems 8(v), 13(v)(vi), and 24.

Theorem 24 also has applications to cylindric algebras. It can be shown that if $\mathfrak{C}$ is a cylindric algebra of dimension 3 or more, and the operation ; is defined on $\mathfrak{C}$ by

$$
x ; y=\left(c_{2} s_{2}^{1} x \cdot s_{2}^{0} y\right)
$$


for all $x, y \in C$, then $\left\langle\mathrm{Nr}_{2} \mathfrak{C}, 1, ;\right\rangle \in \mathrm{GA}$. For a more general result, suppose $\mathfrak{D}$ is a diagonal-free cylindric algebra of dimension 3 or more, $e_{0}$ and $e_{1}$ are two elements of $\mathfrak{D}$ such that $\mathrm{c}_{0} e_{0}=e_{0}$ and $\mathrm{c}_{1} e_{1}=e_{1}$, and the operation; is defined by

$$
x ; y=\left(\mathrm{c}_{2} \mathrm{c}_{1}\left(e_{0} \cdot x\right) \cdot \mathrm{c}_{0}\left(e_{1} \cdot y\right)\right)
$$

for all $x, y \in D$. Then $\left\langle\mathrm{Nr}_{2} \mathfrak{D}, 1, ;\right\rangle \in \mathrm{GA}$. Some of the derivations involved in proving this appear in [Ma81]. The previous result follows from this one when $e_{0}=\mathrm{d}_{12}$ and $e_{1}=\mathrm{d}_{02}$. In both cases we may apply Theorem 24 to obtain a general associativity result. For dimension 4 or more, there is no point in doing this, since the operations defined above are associative when restricted to $\mathrm{Nr}_{2} \mathfrak{C}$ or $\mathrm{Nr}_{2} \mathfrak{D}$. However, they may not be associative for dimension 3. (See 3.2.69 and 5.3.8 of [HMT85].)

\section{IDEAL ELEMENTS, RELATIVIZATIONS, AND HOMOMORPHISMS}

Definition 26. Let $\mathfrak{A} \in \mathrm{NA}$. Then

(i) Ie $\mathfrak{A}=\{x: x \in A, x=x ; 1=1 ; x\}$,

(ii) $\operatorname{Sr} \mathfrak{A}=\left\{x: x \in A, x=\breve{x}=\left(1^{\prime} \cdot x\right) ; x\right\}$.

The elements of Ie $\mathfrak{A}$ are called ideal elements of $\mathfrak{A}$, and the elements of $\operatorname{Sr} \mathfrak{A}$ are called symmetric-reflexive elements of $\mathfrak{A}$.

Ideal elements were originally introduced for relation algebras; see [CT51, Definition 3.23]. The only ideal elements in $\mathfrak{R e} U$ are $\varnothing$ and $U \times U$. To get a less trivial example of ideal elements, let $U$ and $V$ be disjoint nonempty sets. Set $E=(U \times U) \cup(V \times V)$. Then the ideal elements of $\mathfrak{S b} E$ are $\varnothing, U \times U$, $V \times V$, and $(U \times U) \cup(V \times V)$.

Symmetric-reflexive elements are introduced in Definition 5.4 of [Ma82]. They play an important role in the relative representation theorem for WA (Theorem 5.20 of [Ma82]). For any nonempty set $U$, a relation $R$ is in $\operatorname{Sr} \mathfrak{R e} U$ just in case $R$ is symmetric, reflexive over its field, and its field is a (possibly proper) subset of $U$. By a "reflexive relation" we mean one that is reflexive over its field (as in [Me87, p. 6], or [K55, p. 9]). Note that $\varnothing$ is reflexive in this sense.

Definition 27. Let $\mathfrak{A} \in \mathrm{NA}$ and $z \in A$. Then $\mathrm{Rl}_{z} \mathfrak{A}=\{x: z \geq x \in A\}$ and

$$
\mathfrak{R l}_{z} \mathfrak{A}=\left\langle\mathrm{Rl}_{z} \mathfrak{A},+, \cdot,{ }^{-z}, 0, z, ;^{z},{ }^{-z}, 1^{, z}\right\rangle
$$

where, for all $x, y \in A$,

$$
x^{-z}=\bar{x} \cdot z, \quad x ;^{z} y=(x ; y) \cdot z, \quad x^{-z}=\breve{x} \cdot z, \quad 1^{, z}=1^{\prime} \cdot z .
$$

$\mathfrak{R l}_{z} \mathfrak{A}$ is the relativization of $\mathfrak{A}$ to $z$.

Relativization and symmetric-reflexive elements are connected in the following way. Suppose $\mathfrak{A} \in N A$ and $z \in A$. Then $\mathfrak{R I}_{z} \mathfrak{A} \in N A$ iff $z \in \operatorname{Sr} \mathfrak{A}$. Furthermore, if $\mathfrak{A} \in W A$, then $\mathfrak{R l} \mathfrak{A}_{z} \in W A$. Thus WA is closed under relativization to symmetric-reflexive elements. However, even if $\mathfrak{A} \in R R A$ it is 
possible that $\mathfrak{R l}_{z} \mathfrak{A} \notin \mathrm{SA}$. For example, suppose $R \in \operatorname{Sr} \mathfrak{R e} U$. Then $R$ is a symmetric and reflexive binary relation, and $\mathfrak{R l}_{z} \mathfrak{A}$ is a complete and atomic WA, but $R$ is transitive iff $\mathfrak{R l}_{R} \mathfrak{R e} U \in \mathrm{SA}$ iff $\mathfrak{R l}_{R} \mathfrak{R e} U \in \mathrm{RRA}$. (See [Ma82, Theorems 5.5, 5.8, and 5.9].) Finally, WA has a characterization in terms of relativization to symmetric and reflexive relations: every complete and atomic WA is in $\left\{\mathfrak{R l}_{z} \mathfrak{R e} U: z \in \operatorname{Sr} \mathfrak{R e} U\right\}$, and WA $=\mathbf{S}\left\{\mathfrak{R l}_{z} \mathfrak{R e} U: z \in \operatorname{Sr} \mathfrak{R e} U\right\}$ (by Theorems 4.2, 5.13, and 5.19 of [Ma82]).

The next theorem states the connection between ideal elements, relativizations, and homomorphisms for nonassociative relation algebras. It was proved first for relation algebras.

Theorem 28. Let $\mathfrak{A} \in \mathrm{NA}, z \in A$, and, for $x \in A$, let $h(x)=x \cdot z$. Then the following statements are equivalent.

(i) $z \in$ Ie $\mathfrak{A}$,

(ii) $h$ is a homomorphism from $\mathfrak{A}$ onto $\mathfrak{R l}_{z} \mathfrak{A}$,

(ii) for all $x, y \in A, x ; y \cdot z=(x \cdot z) ;(y \cdot z)$.

Proof. The equivalence of parts (i) and (ii) is stated without proof in [CT51, p. 368]. The implication from (i) to (ii) is proved for relation algebras without any use of associativity in [JT52, Theorem 4.9].

The implication from (ii) to (iii) is trivial.

The equivalence of (i) and (iii) follows from that part of Theorem 4.1 of [CT51] whose proof has two easily eliminated uses of associativity. (See the remarks prior to Theorem 8 .)

The next theorem is taken from [Ma78a, pp. 103-106] and [JT52, Theorem 4.10]. The equivalence of $(\alpha)$ and $(\gamma)$ for relation algebras is due to McKinsey and Tarski, and is announced without proof in [JT48, result 4]. The equivalence of $(\alpha),(\gamma)$, and $(\delta)$ for relation algebras is also due to McKinsey and Tarski, and is stated without proof in [CT51, p. 362]. The equivalence of $(\alpha),(\beta)$, $(\gamma)$, and $(\delta)$ is proved for relation algebras in Theorem 4.10 of [JT52]. The part of that proof which shows $(\alpha)$ and $(\beta)$ are equivalent uses only those theorems from [CT51] which are proved without associativity. We therefore omit the proof of part (i) below. As we shall see, the remaining equivalences fail for some WA. Hence the proof in [JT52] makes essential use of associativity, but for each such use the semiassociative law suffices.

Theorem 29. Let $\mathfrak{A} \in$ NA. Consider the following four conditions on $\mathfrak{A}$ :

( $\alpha$ ) for every $x \in A$, if $x \neq 0$ then $1=1 ; x ; 1$,

( $\beta$ ) for all $x, y \in A$, if $0=x ; 1 ; y$ then $x=0$ or $y=0$,

$(\gamma) \mathfrak{A}$ is simple or $0=1$,

( $\delta)$ Ie $\mathfrak{A}=\{0,1\}$.

(i) Conditions $(\alpha)$ and $(\beta)$ are equivalent.

(ii) Conditions $(\alpha)$ and $(\beta)$ imply $(\gamma)$, and $(\gamma)$ implies $(\delta)$.

(iii) If $\mathfrak{A}$ is finite, then $(\gamma)$ and $(\delta)$ are equivalent.

(iv) If $\mathfrak{A} \in \mathrm{SA}$, then $(\alpha),(\beta),(\gamma)$, and $(\delta)$ are equivalent. 
(v) It is not possible to replace "SA" with "WA" in part (iv). In fact, none of the implications beyond the ones stated in (i)-(iii) hold in general for WA's.

Proof. (ii): Assume $(\alpha)$ holds. To prove $(\gamma)$, we assume $0 \neq 1$ and show $\mathfrak{A}$ is simple. Suppose $h$ is a homomorphism of $\mathfrak{A}$ onto some $\mathfrak{B} \in \mathrm{NA}$, and $h$ is not an isomorphism. Then there is some $x \in A$ such that $x \neq 0$ and $h(x)=0$. By $(\alpha), 1=1 ; x ; 1$, so $1=h(1)=h(1 ; x ; 1)=h(1) ; h(x) ; h(1)=1 ; 0 ; 1=0$, i.e., $\mathfrak{B}$ is the one-element algebra. Thus $\mathfrak{A}$ is simple.

Next, assume $(\gamma)$. We have $0,1 \in$ Ie $\mathfrak{A}$ by Theorem 6(ii) and Theorem $8(\mathrm{v})$, so for $(\delta)$ it will suffice to show that Ie $\mathfrak{A}$ has no elements other than 0 and 1. Suppose that $z \neq 1$ and $z \in$ Ie $\mathfrak{A}$. Let $h(x)=x \cdot z$ for every $x \in \mathfrak{A}$. Then $h$ is a homomorphism on $\mathfrak{A}$ by Theorem 28 , and $h$ is not an isomorphism since $\bar{z} \neq 0$ and $h(\bar{z})=0$. Now $0 \neq 1$ since $z \neq 1$, so $\mathfrak{A}$ is simple by $(\gamma)$, and therefore $h$ must map $\mathfrak{A}$ onto a one-element algebra. Hence $z=h(1)=h(0)=0$. Thus $(\gamma)$ implies $(\delta)$.

(iii): Assume $\mathfrak{A}$ is finite and $(\delta)$ holds, i.e., Ie $\mathfrak{A}=\{0,1\}$. If $0=1$ then $(\gamma)$ holds, so assume $0 \neq 1$. Let $h$ be a homomorphism on $\mathfrak{A}$. Let

$$
I=\{x: x \in A, h(x)=0\} \quad \text { and } \quad z=\sum I .
$$

Note that $I$ is finite since $\mathfrak{A}$ is finite, and $I$ is nonempty since $0 \in I$. Then

$$
h(z)=h\left(\sum I\right)=\sum\{h(x): x \in I\}=\sum\{0: x \in I\}=0,
$$

hence $h(z ; 1)=h(z) ; h(1)=0 ; h(1)=0$, so $z ; 1 \in I$. Consequently, $z ; 1 \leq$ $\sum I=z$, but $z \leq z ; 1$ by Theorem 8 (iv), so $z=z ; 1$. Similarly, $z=1 ; z$, so $z \in \operatorname{Ie} \mathfrak{A}$. By $(\delta), z=0$ or $z=1$. It also follows from (1) and (2) that $h(x)=0$ iff $x \leq z$. So, if $z=1$, then $h$ maps $\mathfrak{A}$ onto a one-element algebra, while if $z=0$, then $h$ is an isomorphism. Therefore $\mathfrak{A}$ is simple.

(iv): In view of parts (i) and (ii), it will suffice to show $(\delta)$ implies $(\alpha)$ whenever $\mathfrak{A} \in \mathrm{SA}$. Accordingly, assume Ie $\mathfrak{A}=\{0,1\}$. To prove $(\alpha)$, assume $0 \neq x \in A$. Then $0 \neq x \leq 1 ; x ; 1$, but $1 ; x ; 1 \in$ Ie $\mathfrak{A}$ by Theorem 13(vii), so $1 ; x ; 1=1$.

(v): For every $n \leq \omega$, let $S(n)=\{\langle i, j\rangle: i, j<n$ and $|i-j| \leq 1\}$, and set $\mathfrak{B}_{n}=\mathrm{Rl}_{S(n)} \mathfrak{R e} n$. Clearly $S(n)$ is a symmetric and reflexive relation, so $S(n) \in \operatorname{Sr} \mathfrak{R e} n$, and $\mathfrak{B}_{n} \in \mathrm{WA} \sim \mathrm{SA}$ when $3 \leq n$ by Theorem 5.9(2) of [Ma82]. $\mathfrak{B}_{n}$ is obviously complete and atomic, since its universe consists of all subsets of $S(n)$. We shall see that if $3 \leq n<\omega$, then $\mathfrak{B}_{n}$ is finite and satisfies $(\gamma)$ but not $(\alpha)$ or $(\beta)$, and $\mathfrak{B}_{\omega}$ satisfies $(\delta)$ but not $(\gamma)$.

Let $R \subseteq S(n)$ and suppose $R \in$ Ie $\mathfrak{B}_{n}$. If $\langle i, j\rangle \in R$ then $\langle i, i\rangle \in R$ since $\langle i, i\rangle \in\{\langle i, j\rangle\}|\{\langle j, i\rangle\} \subseteq R| S(n)=R$, and $\langle j, j\rangle \in R$ since $\langle j, j\rangle \in$ $\{\langle j, i\rangle\}|\{\langle i, j\rangle\} \subseteq S(n)| R=R$. Also, if $\langle i, i\rangle \in R$, and $\langle i, j\rangle \in S(n)$, then $\langle i, j\rangle \in R$ since $\langle i, j\rangle \in\{\langle i, i\rangle\}|\{\langle i, j\rangle\} \subset R| S(n)=R$. It follows that $\varnothing$ and $S(n)$ are the only ideal elements of $\mathfrak{B}_{n}$, so $\mathfrak{B}_{n}$ satisfies $(\delta)$. If $n<\omega$, then $\mathfrak{B}_{n}$ is finite but nontrivial, and hence is simple by part 
(iii). But $(\alpha)$ fails in $\mathfrak{B}_{n}$ because $\{\langle 0,0\rangle\} \neq \varnothing$, while $S(n)|\{\langle 0,0\rangle\}| S(n)=$ $\{\langle 0,0\rangle,\langle 0,1\rangle,\langle 1,0\rangle,\langle 1,1\rangle\} \neq S(n)$ since $n \geq 3$.

Finally, $\mathfrak{B}_{\omega}$ is not simple, since there is a nontrivial congruence relation $\equiv$ on $\mathfrak{B}_{\omega}$, which is defined as follows: $R \equiv T$ iff $R, T \subseteq S(\omega)$ and $|(R \sim T) \cup(T \sim R)|<\omega$. Obviously $\equiv$ is an equivalence relation. The proof that $\equiv$ is a congruence relation is fairly straightforward. We will consider just one case.

Suppose $R \equiv T$ and $R^{\prime} \equiv T^{\prime}$. Then $R \sim T, T \sim R, R^{\prime} \sim T^{\prime}$, and $T^{\prime} \sim R^{\prime}$ are finite. To show $R\left|R^{\prime} \equiv T\right| T^{\prime}$, we must show $\left(R \mid R^{\prime}\right) \sim\left(T \mid T^{\prime}\right)$ and $\left(T \mid T^{\prime}\right) \sim\left(R \mid R^{\prime}\right)$ are finite. First note that

$$
\begin{aligned}
\left(R \mid R^{\prime}\right) \sim\left(T \mid T^{\prime}\right) & \subseteq\left(R \mid\left(R^{\prime} \sim T^{\prime}\right)\right) \cup\left((R \sim T) \mid R^{\prime}\right) \\
& \subseteq\left(S(\omega) \mid\left(R^{\prime} \sim T^{\prime}\right)\right) \cup((R \sim T) \mid S(\omega))
\end{aligned}
$$

(more generally, every NA satisfies the identity $w ; x \cdot(y ; z)^{-} \leq w ;(x \cdot \bar{z})+$ $(w \cdot \bar{y}) ; x)$. Now $S(\omega) \mid\left(R^{\prime} \sim T^{\prime}\right)$ and $(R \sim T) \mid S(\omega)$ are finite, because $R^{\prime} \sim T^{\prime}$ and $R \sim T$ are finite and every $\kappa \in \omega$ has finite $S(\omega)$-degree. Hence $\left(R \mid R^{\prime}\right) \sim$ $\left(T \mid T^{\prime}\right)$ is also finite, and so is $\left(T \mid T^{\prime}\right) \sim\left(R \mid R^{\prime}\right)$, for similar reasons.

\section{REPRESENTABILITY AND RELATIONAL BASES}

In this section we prove that certain relation algebras are representable iff they are completely representable (Theorem 33), and that a NA is representable iff it can be embedded in an atomic NA with an $\omega$-dimensional relational basis (Theorem 37).

Definition 30. For any $\mathfrak{A} \in \mathrm{NA}, \mathrm{Eq} \mathfrak{A}=\{x: x ; x \leq \breve{x}=x \in A\}$. Eq $\mathfrak{A}$ is the set of all equivalence elements of $\mathfrak{A}$.

An equivalence relation is just a symmetric and transitive relation (as in [CT51, p. 345] or [HMT71, p. 30]). Every equivalence relation is reflexive and hence is an equivalence relation on its field. For any equivalence relation $E$, $\mathrm{Eq} \mathfrak{S b} E$ is the set of equivalence relations contained in $E$. The field of any $R \in \mathrm{Eq} \mathfrak{S b} E$ may be a proper subset of the field of $E$. In particular, for any set $U, R \in \mathrm{Eq} \mathfrak{R e} U$ just in case $R$ is an equivalence relation over some (possibly proper) subset of $U$. Note that $\varnothing$ is such a relation.

The following theorem is noted in a remark preceding Theorem 4.10 in [JT52].

Theorem 31. If $\mathfrak{A} \in \mathrm{RA}$ and $z \in \mathrm{Eq} \mathfrak{A}$, then $\mathfrak{R l}_{z} \mathfrak{A} \in \mathrm{RA}$.

The next theorem shows that if $\mathfrak{A}$ is an atomic RA with "finite products", then every embedding of $\mathfrak{A}$ into a complete atomic RA $\mathfrak{B}$ can be "relativized" to a complete embedding of $\mathfrak{A}$ into a relativization (and homomorphic image) of $\mathfrak{B}$.

Theorem 32. Assume $\mathfrak{A} \in \mathrm{RA}, \mathfrak{A}$ is atomic, and $\{w: x ; y \geq w \in \mathrm{At} \mathfrak{A}\}$ is finite for all $x, y \in \mathrm{At} \mathfrak{A}$. Suppose $\mathfrak{B}$ is a complete atomic RA and $h$ is an embedding 
of $\mathfrak{A}$ into $\mathfrak{B}$. Let $z=\sum^{(\mathfrak{B})} h^{*}$ At $\mathfrak{A}$, and, for all $x \in A, f(x)=z \cdot h(x)$. Then $z \in \mathrm{Eq} \mathfrak{B}, \mathfrak{R l}_{z} \mathfrak{B}$ is a complete atomic $\mathrm{RA}$, and $f$ is a complete embedding of $\mathfrak{A}$ into $\mathfrak{R l}{ }_{z} \mathfrak{B}$.

Proof. First we have

$$
\begin{array}{rlrl}
\breve{z} & =\left(\sum^{(\mathfrak{B})} h^{*} \text { At } \mathfrak{A}\right)^{\smile} & \\
& =\sum^{(\mathfrak{B})}\left\{(h(y))^{\smile}: y \in \text { At } \mathfrak{A}\right\} & & ; \text { is completely additive } \\
& =\sum^{(\mathfrak{B})}\{h(\breve{y}): y \in \text { At } \mathfrak{A}\} & & h \text { is a homomorphism } \\
& \leq \sum^{(\mathfrak{B})} h^{*} \text { At } \mathfrak{A} & & \text { Theorem 10(i) } \\
& =z, & &
\end{array}
$$

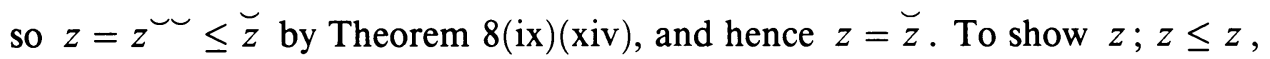
assume $z ; z \geq u \in A t \mathfrak{B}$. By the definition of $z$ and the complete additivity of ;, we have

$$
\begin{aligned}
z ; z & =\sum\{h(x) ; h(y): x, y \in \text { At } \mathfrak{A}\} \\
& =\sum\{h(x ; y): x, y \in \text { At } \mathfrak{A}\} .
\end{aligned}
$$

Hence there are $x, y \in$ At $\mathfrak{A}$ such that $u \leq h(x ; y)$. By the assumptions concerning $\mathfrak{A}$, there are finitely many atoms $v_{0}, \ldots, v_{n-1}$ such that $x ; y=$ $v_{0}+\cdots+v_{n-1}$, so

$$
u \leq h\left(v_{0}+\cdots+v_{n-1}\right)=h\left(v_{0}\right)+\cdots+h\left(v_{n-1}\right) .
$$

Hence, for some $m<n, u \leq h\left(v_{m}\right)$ and $v_{m} \in$ At $\mathfrak{A}$, i.e., $u \leq z$. Thus $z ; z \leq z$. From this and $\breve{z}=z$ we get $z \in \mathrm{Eq} \mathfrak{B}$, and hence $\mathfrak{R l}_{z} \mathfrak{B} \in \mathrm{RA}$ by Theorem 31 .

To show $f$ is a homomorphism we must show, for all $x, y \in A$ :

(1) $f(x+y)=f(x)+f(y)$,

(2) $f(x \cdot y)=f(x) \cdot f(y)$,

(3) $f(\bar{x})=(f(x))^{-z}$,

(4) $f(0)=0$ and $f(1)=z$,

(5) $f(x ; y)=f(x) ;^{z} f(y)$,

(6) $f(\bar{x})=(f(x))^{-z}$,

(7) $f\left(1^{\prime}\right)=1^{, z}$.

Now (1)-(4) and (7) follow by Boolean algebra from the fact that $h$ is a homomorphism. For (6) we have

$$
f(\breve{x})=z \cdot h(\breve{x})=z \cdot \breve{z} \cdot h(\breve{x})=z \cdot(z \cdot h(x))^{\smile}=z \cdot(f(x))^{\smile}=(f(x))^{\smile z} .
$$

For the proof of $(5)$, first note that

$$
\begin{aligned}
f(x) ; f(y) & =(z \cdot h(x)) ;(z \cdot h(y)) \\
& \leq z ; z \cdot h(x) ; h(y) \leq z \cdot h(x ; y)=f(x ; y) .
\end{aligned}
$$


For the opposite inclusion, we begin by assuming $f(x ; y) \geq u \in$ At $\mathfrak{B}$. Then $u \leq f(x ; y)=z \cdot h(x ; y) \leq z=\sum h^{*}$ At $\mathfrak{A}$ so there is some $w \in$ At $\mathfrak{A}$ such that $u \leq h(w)$. Therefore, $0 \neq u \leq h(w) \cdot h(x ; y)=h(w \cdot x ; y)$, so $0 \neq w \cdot x ; y$ and $w \leq x ; y$. By the complete additivity of ; we have

$$
x ; y=\sum^{(\mathfrak{A})}\{q ; r: x \geq q \in \operatorname{At} \mathfrak{A}, y \geq r \in \operatorname{At} \mathfrak{A}\},
$$

so there must be $q, r \in$ At $\mathfrak{A}$ such that $w \leq q ; r \leq x ; y, q \leq x$, and $r \leq y$. Therefore $h(q) \leq z \cdot h(x)=f(x)$ and $h(r) \leq z \cdot h(y)=f(y)$, so $u \leq h(w) \leq$ $h(q ; r)=h(q) ; h(r) \leq f(x) ; f(y)$. Thus any atom of $\mathfrak{B}$ below $f(x ; y)$ is also below $f(x) ; f(y)$, i.e., $f(x ; y) \leq f(x) ; f(y)$. This completes the proof of $(1)-(7)$.

If $x \in$ At $\mathfrak{A}$, then $h(x) \neq 0$ since $h$ is one-to-one, and $h(x) \leq z$ by the definition of $z$, so $f(x)=z \cdot h(x)=h(x) \neq 0$. Thus $f$ is one-to-one.

To show $f$ is complete, assume $X \subseteq A$ and $w=\sum^{(\mathfrak{A})} X$ exists. We must show $\sum^{(\mathfrak{B})} f^{*} X=f(w)$.

Suppose $f(x)$ is an arbitrary element of $f^{*} X$, with $x \in X$. Then $x \leq w$ and $f(x) \leq f(w)$ since $f$ is a homomorphism. Thus $\sum^{(\mathfrak{B})} f^{*} X \leq f(w)$.

For the opposite inclusion, assume $f(w) \geq u \in$ At $\mathfrak{B}$. Then $u \leq f(w) \leq z$, so there is some $y \in \operatorname{At} \mathfrak{A}$ such that $u \leq h(y)$. Then $0 \neq h(y) \cdot f(w) \leq$ $h(y) \cdot h(w)=h(y \cdot w)=h\left(\sum^{(\mathfrak{A})}\{y \cdot x: x \in X\}\right)$, so $0 \neq \sum^{(\mathfrak{A})}\{y \cdot x: x \in X\}$. Since $y \in$ At $\mathfrak{A}$, there must be some $x \in X$ such that $y \leq x$. Hence $u \leq h(y) \leq h(x)$, and we have $u \leq z$, so $u \leq f(x)$ and $x \in X$, i.e., $u \leq \sum^{(\mathfrak{B})} f^{*} X$. Thus $f(w) \leq \sum^{(\mathfrak{B})} f^{*} X$, which finishes the proof that $f$ is complete.

It is not true that a representable relation algebra need be completely representable. In fact, the first example of an infinite representable relation algebra which is not completely representable appears in [L50]. There are other examples of such algebras in [Ma78a]. Thus, the problem arises of finding partial criteria under which representability implies complete representability. The following theorem gives one such criterion.

Theorem 33. Assume $\mathfrak{A} \in \mathrm{RA}, \mathfrak{A}$ is atomic, and $\{w: x ; y \geq w \in$ At $\mathfrak{A}\}$ is finite for all $x, y \in \mathrm{At} \mathfrak{A}$. Then $\mathfrak{A}$ is representable iff $\mathfrak{A}$ is completely representable.

Proof. Let $h$ be a representation of $\mathfrak{A}$ over the equivalence relation $E$. Note that $\mathfrak{S b} E$ is a complete atomic RA and $h$ is an embedding of $\mathfrak{A}$ into $\mathfrak{S b} E$. By Theorem 32 there is a complete isomorphism $f$ mapping $\mathfrak{A}$ into $\mathfrak{R l}_{z} \mathfrak{S b} E$, where $z=\sum^{(\mathfrak{S b} E)} h^{*}$ At $\mathfrak{A}=\bigcup h^{*}$ At $\mathfrak{A} \in \operatorname{EqS} \mathfrak{b} E$, but $\mathfrak{R l}_{z} \mathfrak{S b} E=\mathfrak{S b} z$, so $f$ is a complete representation of $\mathfrak{A}$ over $z$.

An obvious corollary of Theorem 33 is that a finite relation algebra is representable iff it is completely representable.

Now we try to motivate the next definition. Let $U$ be a nonempty set. Let $u \in{ }^{\alpha} U$, where $\alpha<\omega$. Then $u$ determines an $\alpha$-by- $\alpha$ matrix $m$ of atoms of $\mathfrak{R e} U$ as follows: for all $\kappa, \lambda<\alpha, m_{\kappa \lambda}=\left\{\left\langle u_{\kappa}, u_{\lambda}\right\rangle\right\}$. It is then easy to see 
that $m$ has properties $\left(\mathrm{B}_{0}\right)-\left(\mathrm{B}_{2}\right)$ listed in part (i) below. We call such matrices "basic," and let $B_{\alpha} \mathfrak{R e} U$ be the set of all such $\alpha$-dimensional matrices. Let $x, y$ be relations in $\mathfrak{R e}_{\mathfrak{e}} U$. Suppose $m_{\kappa \lambda} \leq x ; y$. Then there is some $v \in U$ such that $\left\langle u_{\kappa}, v\right\rangle \in x$ and $\left\langle v, u_{\lambda}\right\rangle \in y$. Choose $\mu<\alpha$ so that $\mu \neq \kappa, \lambda$. Form the sequence $u^{\prime}$ by replacing $u_{\mu}$ with $v$. Then $u^{\prime}$ "witnesses" the fact that $m_{\kappa \lambda} \leq x ; y$. The sequence $u^{\prime}$ determines another basic matrix $m^{\prime}$ which is the same as $m$ except in row $\mu$ and column $\mu$. The set $B_{\alpha} \mathfrak{R e} U$ thus happens to satisfy the "extension condition" $\left(\mathbf{R}_{2}\right)$ in part (iii) of the definition below, and forms what we call an $\alpha$-dimensional relational basis.

Definition 34. Let $\mathfrak{A} \in N A$ and let $\alpha$ be any ordinal.

(i) $B_{\alpha} \mathfrak{A}$ is the set of $\alpha$-by- $\alpha$ matrices of atoms of $\mathfrak{A}$ which satisfy the following conditions:

$$
\begin{array}{ll}
\left(\mathrm{B}_{0}\right) & m_{\kappa \kappa} \leq 1 \text { ' for all } \kappa<\alpha, \\
\left(\mathrm{B}_{1}\right) & \tilde{m}_{\kappa \lambda}=m_{\lambda \kappa} \text { for all } \kappa, \lambda<\alpha, \\
\left(\mathrm{B}_{2}\right) & m_{\kappa \lambda} \leq m_{\kappa \mu} ; m_{\mu \lambda} \text { for all } \kappa, \lambda, \mu<\alpha .
\end{array}
$$

The elements of $B_{\alpha} \mathfrak{A}$ are called atom matrices or basic matrices.

(ii) The basic matrices $m$ and $m^{\prime}$ in $B_{\alpha} \mathfrak{A}$ agree up to $\kappa$ if $m_{\mu \nu}=m_{\mu \nu}^{\prime}$ whenever $\mu, \nu \in \alpha \sim\{\kappa\}$, and they agree up to $\kappa, \lambda$ if $m_{\mu \nu}=m_{\mu \nu}^{\prime}$ whenever $\mu, \nu \in \alpha \sim\{\kappa, \lambda\}$.

(iii) Let $\alpha \geq 2$. $M$ is an $\alpha$-dimensional relational basis for $\mathfrak{A}$ if $\left(\mathrm{R}_{0}\right) \quad M \subseteq B_{\alpha} \mathfrak{A}$,

$\left(\mathrm{R}_{1}\right)$ for every atom $x \in A t \mathfrak{A}$ there is a basic matrix $m \in M$ such that $m_{01}=x$,

$\left(\mathrm{R}_{2}\right)$ if $m \in M, \kappa, \lambda<\alpha, x, y \in \mathrm{At} \mathfrak{A}, m_{\kappa \lambda} \leq x ; y$, and $\kappa, \lambda \neq \mu<$ $\alpha$, then there is some $m^{\prime} \in M$ such that $m$ and $m^{\prime}$ agree up to $\mu, m_{\kappa \mu}^{\prime}=x$, and $m_{\mu \lambda}^{\prime}=y$.

(iv) $\mathfrak{A}$ is a relation algebra of dimension $\alpha$ if $\mathfrak{A}$ is a subalgebra of some complete atomic nonassociative relation algebra which has an $\alpha$-dimensional relational basis.

(v) $\mathrm{RA}_{\alpha}$ is the class of all relation algebras of dimension $\alpha$.

The notion of relational basis was introduced in [Ma83], where it was called simply "basis." In [Ma83] the notation $\mathrm{MA}_{\alpha}$ was used instead of $\mathrm{RA}_{\alpha}$.

Every basic matrix $m \in B_{\alpha} \mathfrak{A}$ gives rise to a "partial representation" $R$ of $\mathfrak{A}$ over $\alpha \times \alpha$ by the definition $R(x)=\left\{\langle\kappa, \lambda\rangle: \kappa, \lambda<\alpha, m_{\kappa \lambda} \leq x\right\}$, for every $x \in A$. Indeed, $R$ has these properties for all $x, y \in A$ :

$$
\begin{gathered}
R(x+y)=R(x) \cup R(y), \quad R(x \cdot y)=R(x) \cap R(y), \quad R(\bar{x})=(\alpha \times \alpha) \sim R(x), \\
R(0)=\varnothing, \quad R(1)=\alpha \times \alpha, \quad R(\breve{x})=R(x)^{-1}, \quad R(x ; y) \supseteq R(x) \mid R(y) .
\end{gathered}
$$

If $\mathfrak{A}$ is countable and has an $\omega$-dimensional relational basis, then the extension condition $\left(R_{2}\right)$ can be used to progressively alter such a partial representation until the last condition becomes an equality and a full representation is obtained. 
We shall prove that a nonassociative relation is representable iff it is embeddable in an atomic NA with an $\omega$-dimensional relational basis. First we need the following connection between SA's and 3-dimensional relational bases.

Theorem 35. Assume $\mathfrak{A} \in \mathrm{NA}$ and $\mathfrak{A}$ is atomic. Then the following statements are equivalent.

(a) $\mathfrak{A} \in \mathrm{SA}$,

( $\beta$ ) $B_{3} \mathfrak{A}$ is a 3-dimensional relational basis for $\mathfrak{A}$,

$(\gamma) \mathfrak{A}$ has a 3-dimensional relational basis.

Proof. Theorem 4 of [Ma83] states that $(\alpha)$ implies $(\beta)$. Obviously $(\beta)$ implies $(\gamma)$, so we will assume $(\gamma)$ and prove $(\alpha)$.

Let $M$ be a 3-dimensional relational basis for $\mathfrak{A}$. To show $\mathfrak{A} \in \mathrm{SA}$ it is enough to show $x ; 1 ; 1 \leq x ; 1$ for every $x \in A$, since $x ; 1 \leq x ; 1 ; 1$ by Theorem 8(iv).

Suppose

$$
a \leq x ; 1 ; 1 \text { and } a \in \text { At } \mathfrak{A} .
$$

Now $\mathfrak{A}$ is atomic, i.e., every element of $\mathfrak{A}$ is the join of atoms below it, and ; is completely additive, so

$$
\begin{aligned}
x ; 1 ; 1 & =\sum\{b: x \geq b \in \text { At } \mathfrak{A}\} ; \sum \text { At } \mathfrak{A} ; \sum \text { At } \mathfrak{A} \\
& =\sum\{b ; c: x \geq b \in \text { At } \mathfrak{A}, c \in \text { At } \mathfrak{A}\} ; \sum \text { At } \mathfrak{A} \\
& =\sum\{d: b ; c \geq d \in \text { At } \mathfrak{A}, x \geq b \in \text { At } \mathfrak{A}, c \in \text { At } \mathfrak{A}\} ; \sum \text { At } \mathfrak{A} \\
& =\sum\{d ; e: b, c, d, e \in \text { At } \mathfrak{A}, d \leq b ; c, b \leq x\} .
\end{aligned}
$$

Hence there are $b, c, d, e \in \operatorname{At} \mathfrak{A}$ such that

$$
a \leq d ; e, \quad d \leq b ; c, \quad \text { and } b \leq x .
$$

Since $M$ is a relational basis and $a \in \operatorname{At} \mathfrak{A}$, there is some $m \in M$ such that

$$
m_{01}=a \text {. }
$$

Thus $m_{01} \leq d ; e$, so, by the extension condition $\left(\mathbf{R}_{2}\right)$ for relational bases, there is some $m^{\prime} \in M$ such that

$$
m_{02}^{\prime}=d, \quad m_{21}^{\prime}=e, \quad \text { and } m, m^{\prime} \text { agree up to } 2 .
$$

Similarly, $m_{02}^{\prime} \leq b ; c$, so there is some $m^{\prime \prime} \in M$ such that

$$
m_{01}^{\prime \prime}=b, \quad m_{12}^{\prime \prime}=c, \quad \text { and } \quad m^{\prime}, m^{\prime \prime} \text { agree up to } 1 .
$$

Then, by (4) and (5),

$$
m_{00}=m_{00}^{\prime}=m_{00}^{\prime \prime} \leq m_{01}^{\prime \prime} ; m_{10}^{\prime \prime}=b ; \breve{b} .
$$

Note that $\breve{b}$ is also an atom by Theorem 10(i). By (6) and the extension condition there is some $m^{\prime \prime \prime} \in M$ such that

$$
m_{02}^{\prime \prime \prime}=b, \quad m_{20}^{\prime \prime \prime}=\breve{b}, \quad \text { and } \quad m, m^{\prime \prime \prime} \text { agree up to } 2 .
$$


Using (3) and (7) we get

$$
a=m_{01}=m_{01}^{\prime \prime \prime} \leq m_{02}^{\prime \prime \prime} ; m_{21}^{\prime \prime \prime}=b ; m_{21}^{\prime \prime \prime} \leq x ; 1 .
$$

Steps (1)-(8) show that $a \leq x ; 1$ whenever $a \leq x ; 1 ; 1$ and $a \in$ At $\mathfrak{A}$. Since $\mathfrak{A}$ is atomic, this shows $x ; 1 ; 1 \leq x ; 1$.

If $\mathfrak{A} \in \mathrm{NA}$, then any algebra $\mathfrak{B} \in \mathrm{NA}$ which satisfies conditions $(\alpha)-(\delta)$ of the next theorem is called a completion of $\mathfrak{A}$. The next theorem says that every NA has a completion, and gives those few additional properties of completions that we will need later.

Theorem 36. Let $\mathfrak{A} \in \mathrm{NA}$. Then there is some algebra $\mathfrak{B}$ such that

$(\alpha) \mathfrak{A} \subseteq \mathfrak{B}$,

$(\beta) \mathfrak{B}$ is complete,

( $\gamma)$ if $X \subseteq A$ and $\sum^{(\mathfrak{A})} X$ exists, then $\sum^{(\mathfrak{A})} X=\sum^{(\mathfrak{B})} X$,

( $\delta)$ for every $b \in B, b=\sum\{a: b \geq a \in A\}$,

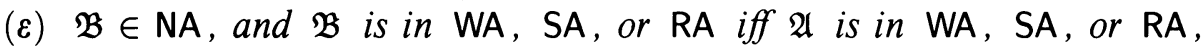
respectively,

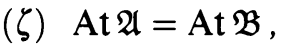

( $\eta$ ) for every $\alpha \geq 2$, if $M$ is an $\alpha$-dimensional relational basis for $\mathfrak{A}$, then $M$ is an $\alpha$-dimensional relational basis for $\mathfrak{B}$.

Proof. By Theorems 1.10, 1.11, and 1.12 of [Ma82], every NA is a Boolean algebra with operators (in the sense of Definition 2.13 of [JT52]) in which the operators $\smile$ and ; are completely additive. So, according to [Mo70, p. 51], there is a Boolean algebra with operators, $\mathfrak{B}$, such that $(\alpha)-(\delta)$ hold. Theorems 1.9 and 1.10 of [Mo70] state that certain types of formulas hold in $\mathfrak{B}$ whenever they hold in $\mathfrak{A}$. The axioms for NA, WA, SA, and RA are of this type, so $\mathfrak{B} \in \mathrm{NA}$ and $(\varepsilon)$ holds. Condition $(\zeta)$ follows from $(\delta)$, while $(\boldsymbol{\eta})$ is an immediate consequence of $(\zeta)$ and the definition of relational basis.

The next theorem is almost equivalent to Theorem 6(3) of [Ma83], which states that $R R A=R A_{\omega}$, i.e., a relation algebra $\mathfrak{A}$ is representable iff $\mathfrak{A}$ can be embedded in a complete atomic SA with an $\omega$-dimensional relational basis. The next theorem shows this is still true if completeness is omitted and "SA" is replaced by "NA".

Theorem 37. Let $\mathfrak{A} \in \mathrm{NA}$. Then the following statements are equivalent.

( $\alpha) \mathfrak{A}$ is representable,

$(\beta)$ there is an atomic $\mathfrak{B} \in \mathrm{NA}$ such that $\mathfrak{A} \subseteq \mathfrak{B}$ and $\mathfrak{B}$ has an $\omega$ dimensional relational basis.

Proof. Assume $\mathfrak{A}$ is representable. Then there is some equivalence relation $E$ and an algebra $\mathfrak{B}$ such that $\mathfrak{A} \subseteq \mathfrak{B} \cong \mathfrak{S} \mathfrak{b} E$. Now $\mathfrak{B}$ is not only atomic, but also complete, and is not only in NA, but also in RRA. Finally, $B_{\omega} \mathfrak{B}$ is a relational basis for $\mathfrak{B}$, since $B_{\omega} \mathfrak{S b} E$ is a relational basis for $\mathfrak{S b} E$. Thus $(\alpha)$ implies $(\beta)$. 
Now assume $(\beta)$ holds, and that $M \subseteq B_{\omega} \mathfrak{B}$ is an $\omega$-dimensional relational basis for $\mathfrak{B}$. Let $\mathfrak{C}$ be a completion of $\mathfrak{B}$, i.e., an algebra obtained by applying Theorem 36 to $\mathfrak{B}$. Then $M$ is also an $\omega$-dimensional relational basis for $\mathfrak{C}$. It is easy to show that $\left\{\left\langle m_{i j}\right\rangle_{i, j<3}: m \in M\right\}$ is a 3-dimensional relational basis for $\mathfrak{C}$, so $\mathfrak{C} \in \mathrm{SA}$ by Theorem 35. Thus $\mathfrak{A} \subseteq \mathfrak{C} \in \mathrm{SA}, \mathfrak{C}$ is complete and atomic, and $\mathfrak{C}$ has an $\omega$-dimensional relational basis. So, by the definition of $\mathrm{RA}_{\omega}$ and Theorem 6(3) of [Ma83], $\mathfrak{A}$ is representable.

Completeness plays no essential role in the proof of Theorem 6(3) of [Ma83]. In fact, it is not even mentioned in the part of the proof which shows RA $\subseteq$ RRA.

\section{IDENTITY ATOMS, POINTS, PAIRS, TWINS, AND FUNCTIONAL ELEMENTS}

In this section we define identity atoms, points, pairs, twins, and functional elements and prove many things about them, including generalizations of some theorems in [J82] and [SS85]. (See Theorems 41 and 42 below.) Except for these generalizations we prove only what we need later. Only one theorem in this section holds for relation algebras but not for semiassociative relation algebras.

Definition 38. For every $\mathfrak{A} \in N A$, let

(i) Ia $\mathfrak{A}=\left\{x: 1^{\prime} \geq x \in\right.$ At $\left.\mathfrak{A}\right\}$,

(ii) Pt $\mathfrak{A}=\{x: 0 \neq x \in A, x ; 1 ; x \leq 1\}$,

(iii) $\operatorname{Pr} \mathfrak{A}=\left\{x: 0 \neq x \in A, x ; 0^{\prime} ; x ; 0^{\prime} ; x \leq 1^{\prime}\right\}$,

(iv) Tw $\mathfrak{A}=\{x: x \in \operatorname{Pr} \mathfrak{A}$ and $x \cdot y=0$ for every $y \in \operatorname{Pt} \mathfrak{A}\}$,

(v) Fn $\mathfrak{A}=\left\{x: x \in A, \breve{x} ; x \leq 1^{\prime}\right\}$.

The elements of Ia $\mathfrak{A}, \operatorname{Pt} \mathfrak{A}, \operatorname{Pr} \mathfrak{A}, \operatorname{Tw} \mathfrak{A}$, and Fn $\mathfrak{A}$ are called the identity atoms, points, pairs, twins, and functional elements, of $\mathfrak{A}$ respectively.

The following observations should explain the intuitive meaning of these definitions. Suppose $\mathfrak{A}$ is a subalgebra of $\mathfrak{R e} U$ and $R$ is a relation in $\mathfrak{A}$.

$R$ is an identity atom iff $R \subseteq \operatorname{Id}_{U}=\{\langle x, x\rangle: x \in U\}, R$ is not empty, and no nonempty proper subrelation of $R$ is an element of $\mathfrak{A}$.

Decoding the definition of point, we see that $R$ is a point just in case $R$ is not empty and every element in the domain of $R$ is the same as every element in the range of $R$, i.e., $R=\{\langle a, a\rangle\}$ for some $a \in U$.

With regard to the definition of pair, notice that, by Theorem 8 (xxvii), every pair is included in the identity. Hence, we can decode the definition of a pair as follows: $R$ is a pair of $\mathfrak{A}$ just in case $R$ is not empty, and whenever $\langle a, a\rangle$, $\langle b, b\rangle,\langle c, c\rangle$ are in $R$ and $b$ is different from $a$ and $c$, then $a=c$, i.e., $R=\{\langle a, a\rangle,\langle b, b\rangle\}$ for some $a, b \in U$.

A twin is a pair which is disjoint from every point, so $R$ is a twin just in case there are distinct $a, b \in U$ such that $R=\{\langle a, a\rangle,\langle b, b\rangle\}$, and neither $\{\langle a, a\rangle\}$ nor $\{\langle b, b\rangle\}$ are elements of $\mathfrak{A}$. 
Finally, $R$ is a functional element of $\mathfrak{A}$ iff $R$ is a function whose domain and range are (possibly proper) subsets of $U$. Notice that $\varnothing$ is functional. Functional elements were introduced and extensively studied for relation algebras in [CT51].

Theorem 39. Let $\mathfrak{A} \in \mathrm{NA}$.

(i) If $x \in \operatorname{Pr} \mathfrak{A}$ then $x \leq 1$ '.

(ii) Pt $\mathfrak{A} \cup \mathrm{Tw} \mathfrak{A} \subseteq \operatorname{Pr} \mathfrak{A}$.

(iii) $\operatorname{Pt} \mathfrak{A} \cap \mathrm{Tw} \mathfrak{A}=\varnothing$.

Proof. (i): This part follows from Theorem 8 (xxvi)(xxvii).

(ii): Trivially, Tw $\mathfrak{A} \subseteq \operatorname{Pr} \mathfrak{A}$. Suppose $x \in \operatorname{Pt} \mathfrak{A}$. Then $x ; 0 ; x=0$ by Theorem 8 (xxvi), so $x ; 0^{\prime} ; x ; 0^{\prime} ; x=0 ; 0^{\prime} ; x=0 \leq 1^{\prime}$, and therefore $x \in$ $\operatorname{Pr} \mathfrak{A}$.

(iii): If $x$ were both a point and a twin, then $x$ would be both nonzero and disjoint from itself.

Theorem 4.6(vii) of [JT52] states that if $\mathfrak{A} \in \mathrm{RA}, x \in$ At $\mathfrak{A}$, and $y \in$ Fn $\mathfrak{A}$, then either $x ; y=0$ or $x ; y \in$ At $\mathfrak{A}$. This result does not hold for every $\mathfrak{A} \in \mathrm{SA}$, but one of its consequences does hold for all SA's, as shown by the first part of the next theorem. The other parts of the theorem contain essential properties of points, pairs, and twins which we will use later.

Theorem 40. Let $\mathfrak{A} \in \mathrm{SA}$.

(i) If $x^{d} \in$ At $\mathfrak{A}$ and $x \in$ Fn $\mathfrak{A}$ then $x \in$ At $\mathfrak{A}$.

(ii) If $\mathfrak{A}$ is simple then $\operatorname{Pr} \mathfrak{A} \sim \operatorname{Ia} \mathfrak{A}=\{u+v: u, v \in \operatorname{Pt} \mathfrak{A}, u \cdot v=0\}$.

(iii) If $\mathfrak{A}$ is simple then $\operatorname{Pr} \mathfrak{A} \cap \mathrm{Ia} \mathfrak{A}=\operatorname{Pt} \mathfrak{A} \cup \operatorname{Tw} \mathfrak{A}$.

(iv) If $x \in \operatorname{Pt} \mathfrak{A}$ and $y \in \operatorname{At} \mathfrak{A}$, then $x ; 1 ; y, y ; 1 ; x \in \operatorname{At} \mathfrak{A} \cup\{0\}$ and $y ; 1 ; x \in$ Fn $\mathfrak{A}$. If $\mathfrak{A}$ is simple then $x ; 1 ; y, y ; 1 ; x \in$ At $\mathfrak{A}$.

(v) If $\mathfrak{A}$ is simple, $x \in \mathrm{Pt} \mathfrak{A}$, and $y \in \operatorname{Pt} \mathfrak{A} \cup \mathrm{Tw} \mathfrak{A}$, then $x ; 1 ; y, y ; 1 ; x \in$ At $\mathfrak{A}$ and $y ; 1 ; x \in$ Fn $\mathfrak{A}$.

(vi) Parts (i), (iii), (iv), and (v) fail in some finite simple WA.

Proof. (i): Suppose $0 \neq z \leq x$. We will show $x \leq z$. First note that $z^{d} \leq x^{d}$ by Theorem 8 (iii)(ix). Furthermore $z^{d} \neq 0$ since; is normal and $0 \neq z=z^{d} ; z$ by Theorem 8 (xxiv). Hence $z^{d}=x^{d}$ since $x^{d} \in$ At $\mathfrak{A}$ by Theorem 12(i). Then

$$
x=x^{\mathrm{d}} ; x=z^{\mathrm{d}} ; x=(1 \cdot z ; \breve{z}) ; x \leq z ; 1 ; 1=z ; 1
$$

by Theorem 8 (iii)(xxiv) and the SL, so

$$
x=x \cdot z ; 1 \leq z ;(1 \cdot \breve{z} ; x) \leq z ;(\widetilde{x} ; x) \leq z ; 1^{\prime}=z
$$

by Theorem 8 (iii)(vi)(ix), $\breve{x} ; x \leq 1$, and the IL.

(ii): Let $x \in \operatorname{Pr} \mathfrak{A} \sim$ Ia $\mathfrak{A}$. By Theorem 39(i), $x \notin$ At $\mathfrak{A}$, so $x=u+v$, $u \cdot v=0$, and $u, v \neq 0$ for some $u, v \in A$. By Theorem 39(i), we have $u, v \leq 1$ '. Hence $u ; 1 ; v=u ; 0^{\prime} ; v$ and $v ; 1 ; u=v ; 0$ ' $u$ by Theorem 
8 (xviii). We also have $1=1 ; u ; 1=1 ; v ; 1$ since $\mathfrak{A}$ is simple, by Theorem 29(iv). Then

$$
\begin{aligned}
u ; 1 ; u & =u ;(1 ; v ; 1) ; u \\
& =u ; 1 ;(v ; 1 ; u) \quad \text { Theorem } 25 \\
& =u ; 1 ;\left(v ; 0^{\prime} ; u\right) \\
& =u ; 1 ; v ; 0^{\prime} ; u \quad \text { Theorem } 25 \\
& =u ; 0^{\prime} ; v ; 0^{\prime} ; u \\
& \leq x ; 0^{\prime} ; x ; 0^{\prime} ; x \\
& \leq 1^{\prime} .
\end{aligned}
$$

Thus $u \neq 0$ and $u ; 1 ; u \leq 1$, so $u \in \operatorname{Pt} \mathfrak{A}$. Similarly, $v \in \operatorname{Pt} \mathfrak{A}$.

For the opposite inclusion, suppose $u, v \in \operatorname{Pt} \mathfrak{A}$ and $u \cdot v=0$. Let $x=$ $u+v$. Clearly $x \notin$ Ia $\mathfrak{A}$ since $u, v \neq 0$. We must show $x ; 0 ; x ; 0 ; x \leq$ 1'. By Theorem 8(i)(ii), $x$ is the join of the elements $r ; 0$ '; $s ; 0$ '; $t$ where $r, s, t \in\{u, v\}$. For such an element, if $r=s$, then $r ; 0^{\prime} ; s ; 0^{\prime} ; t=0 ; 0^{\prime} ; t=$ 0 by Theorem 8 (xxvi), and if $s=t$, then $r ; 0^{\prime} ; s ; 0^{\prime} ; t \leq 1 ; s ; 0^{\prime} ; t=$ $1 ;\left(s ; 0^{\prime} ; t\right)=1 ; 0=0$ by Theorems 8 (iii), 25 , and 8 (xxvi), so $x ; 0^{\prime} ; x ; 0^{\prime} ; x$ $=u ; 0^{\prime} ; v ; 0^{\prime} ; u+v ; 0^{\prime} ; u ; 0^{\prime} ; v \leq u ; 1 ; 1 ; 1 ; u+v ; 1 ; 1 ; 1 ; v=u ; 1 ; u+$ $v ; 1 ; v \leq 1$ ' by the SL.

(iii): First we show Pt $\mathfrak{A} \subseteq$ Ia $\mathfrak{A}$. Let $x \in \operatorname{Pt} \mathfrak{A}$. Then $1^{\prime} \geq x \neq 0=x ; 0$ ' $; x$ by Theorem 8 (xxvi). For every $y \in A,(x \cdot y) ; 1 ;(x \cdot \bar{y})=(x \cdot y) ; 0$ ' $(x \cdot \bar{y}) \leq$ $x ; 0 ; x=0$ by Theorem 8 (xviii), hence $x \cdot y=0$ or $x \cdot \bar{y}=0$, by Theorem 29(iv). Therefore $x \in$ Ia $A$.

If $x \in \mathrm{Tw} \mathfrak{A}$ then $x$ is a pair disjoint from every point. In particular, $x$ is not the join of two points, so it must be an atom by part (ii). Thus Tw $\mathfrak{A} \subseteq$ Ia $\mathfrak{A}$.

Combining what has been proved so far with Theorem 39(ii) yields $\operatorname{Pr} \mathfrak{A} \cap$ Ia $\mathfrak{A} \supseteq \operatorname{Pt} \mathfrak{A} \cup$ Tw $\mathfrak{A}$.

For the opposite inclusion, we assume $x \in \operatorname{Pr} \mathfrak{A} \cap \operatorname{Ia} \mathfrak{A} \sim \operatorname{Tw} \mathfrak{A}$ and show $x \in \operatorname{Pt} \mathfrak{A}$. Since $x \in \operatorname{Pr} \mathfrak{A} \sim \operatorname{Tw} \mathfrak{A}$, there is some $y \in \operatorname{Pt} \mathfrak{A}$ such that $x \cdot y \neq 0$. Hence $y \leq x$ since Pt $\mathfrak{A} \subseteq$ Ia $\mathfrak{A}$. But $x \in$ Ia $\mathfrak{A}$, so $x=y \in \operatorname{Pt} \mathfrak{A}$.

(iv): We will assume $x ; 1 ; y \neq 0$ and show $x ; 1 ; y \in$ At $\mathfrak{A}$. Suppose $0 \neq z$. $x ; 1 ; y$. Then $0 \neq y \cdot(x ; 1)^{\smile} ; z=y \cdot 1 ; x ; z$ by Theorems $8(\mathrm{xii})(\mathrm{xiii})(\mathrm{xv})(\mathrm{xxvi})$, and the cycle law. Since $y \in$ At $\mathfrak{A}$, this yields $y \leq 1 ; x ; z$. Then $x ; 1 ; y \leq$ $x ; 1 ;(1 ; x ; z)=x ; 1 ; x ; z \leq 1 ' ; z \leq z$ by Theorems $8($ iii)(v), 25, (xxvi) and the IL. Thus $x ; 1 ; y \in$ At $\mathfrak{A}$, and, similarly, $y ; 1 ; x \in$ At $\mathfrak{A} \cup\{0\}$. By Theorems 8(iii)(xii)(xiii)(xv)(xxvi) and, 25,

$$
\begin{aligned}
(y ; 1 ; x)^{\smile} ;(y ; 1 ; x) & =x ;(1 ; \breve{y}) ;(y ; 1 ; x) \\
& =x ;(1 ; \breve{y} ; y ; 1) ; x \leq x ; 1 ; x \leq 1,
\end{aligned}
$$

so $y ; 1 ; x \in$ Fn $\mathfrak{A}$.

Now suppose $\mathfrak{A}$ is simple. Since $x \neq 0 \neq y$, we get $x ; 1 ; y \neq 0 \neq y ; 1 ; x$ by Theorem 29 (iv). Hence $x ; 1 ; y, y ; 1 ; x ; \in$ At $\mathfrak{A}$. 
(v): We have $y \in$ At $\mathfrak{A}$ by part (iii), so the desired conclusion follows by part (iv).

(vi): Let $\mathfrak{A}=\mathfrak{R l}_{S(3)} \mathfrak{R e} 3$, where $S(3)=\{\langle i, j\rangle: i, j<3$ and $|i-j| \leq 1\}$. Then $\mathfrak{A}$ is a simple finite WA by [Ma82, 5.9(2)].

To show part (i) fails, let $x=\{\langle 1,2\rangle,\langle 1,0\rangle\}$. Then $x^{d}=\{\langle 1,1\rangle\} \in$ At $\mathfrak{A}$ and $\breve{x} ; x=\{\langle 0,0\rangle,\langle 2,2\rangle\} \leq 1$, so $x \in$ Fn $\mathfrak{A}$, but $x \notin$ At $\mathfrak{A}$.

To show parts (iii)-(v) fail, let $x=\{\langle 0,0\rangle,\langle 2,2\rangle\}$ and $y=\{\langle 1,1\rangle\}$. Then $x \in \operatorname{Pt} \mathfrak{A}$ but $x \notin$ At $\mathfrak{A}$, so (iii) fails. Also $y \in$ At $\mathfrak{A} \cap \operatorname{Pt} \mathfrak{A}$ but $x ; 1 ; y=$ $\{\langle 0,1\rangle,\langle 2,1\rangle\} \notin$ At $\mathfrak{A} \cup\{0\}$, so (iv) and (v) fail.

Now we obtain two theorems which improve previously published results.

Theorem 41. If $\mathfrak{A} \in \mathrm{SA}, \mathfrak{A}$ is simple, $0 \neq x \in A, x ; 1 ; \bar{x} \leq 1$, and $\breve{x} ; 1 ; x \leq$ 1 , then $x \in \operatorname{At} \mathfrak{A}$ and $x^{\mathrm{d}}, x^{\mathrm{r}} \in \operatorname{Pt} \mathfrak{A}$.

Proof. By Theorems 8(iii) and 25, we have

$$
x^{\mathrm{d}} ; 1 ; x^{\mathrm{d}} \leq x ; \breve{x} ; 1 ;(x ; \breve{x}) \leq x ;(\breve{x} ; 1 ; x) ; \breve{x} \leq x ; 1 ; \breve{x} \leq 1,
$$

and $x^{d} \neq 0$ since $x \neq 0$, so $x^{d} \in \operatorname{Pt} \mathfrak{A}$. Similarly, $x^{r} \in \operatorname{Pt} \mathfrak{A}$. By Theorem $40(\mathrm{v}), x^{\mathrm{d}} ; 1 ; x^{r} \in$ At $\mathfrak{A}$. But, by Theorem 8(iii)(xxiv), $0 \neq x=x^{\mathrm{d}} ; x ; x^{\mathrm{r}} \leq$ $x^{\mathrm{d}} ; 1 ; x^{r} \in$ At $\mathfrak{A}$, so $x=x^{\mathrm{d}} ; 1 ; x^{r}$ and $x \in$ At $\mathfrak{A}$.

Theorem 41, restricted to relation algebras and without the conclusion that $x^{\mathrm{d}}, x^{\mathrm{r}} \in \mathrm{Pt} \mathfrak{A}$, is related to Theorem 4.30 of [JT52]. Theorem 41 shows that if the RA $\mathfrak{A}$ in part (ii) of Theorem 4.30 is simple, then $\mathfrak{A}$ is also atomic, and therefore "atomistic" may be deleted from part (ii) if "simple" is added. Jónsson and Tarski knew that Theorem 41 holds for RA's, but did not state it in [JT52]. It was first published as Lemma 7.3 in [J82], and is generalized here to SA's.

Theorem 42. If $\mathfrak{A} \in \mathrm{SA}, \mathfrak{A}$ is simple, $x, y \in A, 0 \neq x=x ; 1,0 \neq y=y ; 1$, $0 \neq x, y$, and $\breve{x}, \breve{y} \in$ Fn $A$, then $x ; \breve{y} \in$ At $\mathfrak{A}$.

Proof. Let $z=x ; \breve{y}$. We will see that Theorem 41 applies to $z$. First we have

$$
\begin{aligned}
z ; 1 ; \breve{z} & =x ;(\breve{y} ; 1 ; y) ; \breve{x} & & \text { Theorems } 8(\mathrm{xiv})(\mathrm{xv}), 25 \\
& \leq x ; 1 ; \breve{x} & & \text { Theorem } 8(\mathrm{iii}) \\
& =x ; \breve{x} \leq 1, & &
\end{aligned}
$$

and, similarly, $\breve{z} ; 1 ; z \leq 1$ '. By Theorem 29(iv) $0 \neq x ; 1 ; \breve{y}=x ; \breve{y}=z$ since $x, y \neq 0$ and $x=x ; 1$. Thus $z \in$ At $\mathfrak{A}$ by Theorem 41 .

Theorem 42 was published as Theorem 7(i) of [SS85], but with the following additional assumptions: $\mathfrak{A} \in \mathrm{RA}, \mathfrak{A}$ is complete, and $\mathfrak{A}$ is atomic. Some of the additional assumptions are not immediately apparent from the statement of 
Theorem 7(i) in [SS85], since the authors use the phrase "relation algebra" to refer to a relation algebra which is also complete, atomic, and simple. The proof of Theorem 42 shows that Theorem 7(i) of [SS85] is actually just a consequence of Lemma 7.3 of [J82].

Theorem 43. (i) If $\mathfrak{A} \in \mathrm{RA}, \mathfrak{A}$ is simple, and $x, y \in \mathrm{Tw} \mathfrak{A}$, then exactly one of the following statements holds:

$(\alpha) x ; 1 ; y \in$ At $\mathfrak{A}$,

( $\beta$ ) there are distinct $v, w$ such that $x ; 1 ; y=v+w$, and $v, w, \breve{v}, \breve{w} \in$ At $\mathfrak{A} \cap$ Fn $\mathfrak{A}$.

(ii) Part (i) fails in some simple SA. In fact, for every $\alpha \geq 2$ there is a simple $\mathfrak{A}_{\alpha} \in \mathrm{SA}$ with distinct $x, y \in A_{\alpha}$ such that $\mathrm{Tw} \mathfrak{A}=\{x, y\}, 1^{\prime}=x+y$, and $x ; 1 ; y$ is the join of $\alpha$ atoms, none of which is functional.

Proof. (i) We assume $(\alpha)$ is false and prove $(\beta)$. Thus we have $x ; 1 ; y \notin$ At $\mathfrak{A}$. We also know that $x ; 1 ; y \neq 0$ since $x, y \neq 0$ and $\mathfrak{A}$ is simple, by Theorem 29(iv). Hence there are $v, w \in A$ such that

$$
x ; 1 ; y=v+w, \quad v \cdot w=0, \quad v \neq 0, \quad \text { and } \quad w \neq 0 .
$$

By Theorems 8(viii)(x)(xii)(xiii)(xiv), and 39(i), this implies

$$
y ; 1 ; x=\breve{v}+\breve{w}, \quad \breve{v} \cdot \breve{w}=0, \quad \breve{v} \neq 0, \quad \text { and } \quad \breve{w} \neq 0 .
$$

Next, we show from (1) that $v, w \in \mathrm{Fn} \mathfrak{A}$. We have $1=1 ; y ; 1$ since $\mathfrak{A}$ is simple and $y \neq 0$. So

$$
\begin{aligned}
x & =x^{\mathrm{d}}=1^{\prime} \cdot x ; 1 & & \text { Theorem } 8(\mathbf{x x i})(\mathbf{x x v}), 39(\mathrm{i}) \\
& =1^{\prime} \cdot x ; 1 ; y ; 1 & & \\
& =1^{\prime} \cdot(v+w) ; 1 & & (1) \\
& =1^{\prime} \cdot v ; 1+1^{\prime} \cdot w ; 1 & & \text { Theorem } 8(\mathrm{i}) \\
& =v^{\mathrm{d}}+w^{\mathrm{d}} & & \text { Theorem } 8(\mathbf{x x i}) .
\end{aligned}
$$

But $v^{\mathrm{d}} \neq 0$ and $w^{\mathrm{d}} \neq 0$ since $v, w \neq 0$, and $x \in$ At $\mathfrak{A}$, so $x=v^{\mathrm{d}}=w^{\mathrm{d}}$ by (3). In a similar way we get $y=v^{r}=w^{r}$. Hence, by Theorem $8(\mathrm{xiii})(\mathrm{xv})(\mathrm{xxiv})$,

$$
\breve{v}=y ; \breve{v}, \quad v=x ; v ; y, \quad x \leq w ; \breve{w}, \quad \text { and } \quad w=w ; y .
$$

The statements in (4) were chosen just for the following derivation, which contains the only essential use in this proof of the associative law.

$$
\begin{aligned}
\breve{v} ; v & =y ; \breve{v} ;(x ; v ; y) \\
& \leq y ; \breve{v} ;(w ; \breve{w} ; v ; y) \\
& =y ; \breve{v} ;(w ; y ; \breve{w} ; v ; y) \\
& =y ;(\breve{v} ; w) ; y ;(\breve{w} ; v) ; y
\end{aligned}
$$


We have $w \leq \bar{v}$ since $v \cdot w=0$, so $\breve{v} ; w \leq \breve{v} ; \bar{v} \leq 0$ ' by Theorem 8 (xxviii), and hence $\widetilde{w} ; v \leq 0$ ' by Theorem 8 (xii)(xiv)(xv). Therefore,

$$
\begin{array}{rlrl}
\breve{v} ; v & \leq y ;(\breve{v} ; w) ; y ;(\breve{w} ; v) ; y & & (5) \\
& \leq y ; 0^{\prime} ; y ; 0 ; y & & \\
& \leq 1 & y \in \operatorname{Tw} \mathfrak{A},
\end{array}
$$

$y \in \operatorname{Tw} \mathfrak{A}$,

so $v \in$ Fn $\mathfrak{A}$. By a similar proof, $w \in$ Fn $\mathfrak{A}$. We have $v^{\mathrm{d}}=w^{\mathrm{d}}=x \in$ At $\mathfrak{A}$, so $v, w \in$ At $\mathfrak{A}$ by Theorem $40(\mathbf{i})$. It follows similarly from (2) that $\breve{v}, \breve{w} \in$ Fn $\mathfrak{A} \cap$ At $\mathfrak{A}$.

(ii): Let $\alpha \geq 1$. Let $\mathfrak{A}_{\alpha}$ be the SA determined by the following conditions.

(1) At $\mathfrak{A}_{\alpha}=\left\{a_{0}, a_{00}, a_{1}, a_{11}\right\} \cup\left\{a_{01}^{\kappa}: \kappa<\alpha\right\} \cup\left\{a_{10}^{\kappa}: \kappa<\alpha\right\}$,

(2) $1^{\prime}=a_{0}+a_{1}$,

(3) if $x \in\left\{a_{0}, a_{00}, a_{1}, a_{11}\right\}$ then $\breve{x}=x$,

(4) if $\kappa<\alpha$ then $\left(a_{01}^{\kappa}\right)^{\smile}=a_{10}^{\kappa}$ and $\left(a_{10}^{\kappa}\right)^{\smile}=a_{01}^{\kappa}$,

(5) if $\kappa, \lambda<2, \kappa \neq \lambda$, and $\mu, \nu<\alpha$, then

$$
\begin{aligned}
a_{\kappa} ; a_{\kappa} & =a_{\kappa \kappa} ; a_{\kappa \kappa}=a_{\kappa}, \\
a_{\kappa} ; a_{\kappa \kappa} & =a_{\kappa \kappa} ; a_{\kappa}=a_{\kappa \kappa}, \\
a_{\kappa} ; a_{\kappa \lambda}^{\mu} & =a_{\kappa \lambda}^{\mu} ; a_{\lambda}=a_{\kappa \lambda}^{\mu}, \\
a_{\kappa \kappa} ; a_{\kappa \lambda}^{\mu} & =a_{\kappa \lambda}^{\mu} ; a_{\lambda \lambda}=\sum_{\nu<\alpha} a_{\kappa \lambda}^{\nu}, \\
a_{\kappa \lambda}^{\mu} ; a_{\lambda \kappa}^{\nu} & = \begin{cases}a_{\kappa}+a_{\kappa \kappa} & \text { if } \mu=\nu, \\
a_{\kappa \kappa} & \text { if } \mu \neq \nu,\end{cases}
\end{aligned}
$$

(6) if $x, y \in$ At $\mathfrak{A}_{\alpha}$ and $x ; y$ is not determined by (5), then $x ; y=0$.

If $\alpha=1$, then the second case in the last part of (5) cannot occur, and $\mathfrak{A}_{1}$ is isomorphic to the subalgebra of $\mathfrak{R e}^{4}$ generated by $\{\langle 0,0\rangle,\langle 2,2\rangle\}$, under the isomorphism $h$ which behaves as follows on the atoms of $\mathfrak{A}_{1}$ :

$$
\begin{aligned}
h\left(a_{0}\right) & =\{\langle 0,0\rangle,\langle 2,2\rangle\}, \\
h\left(a_{00}\right) & =\{\langle 0,2\rangle,\langle 2,0\rangle\}, \\
h\left(a_{1}\right) & =\{\langle 1,1\rangle,\langle 3,3\rangle\}, \\
h\left(a_{11}\right) & =\{\langle 1,3\rangle,\langle 3,1\rangle\}, \\
h\left(a_{01}^{0}\right) & =\{\langle 0,1\rangle,\langle 0,3\rangle,\langle 2,1\rangle,\langle 2,3\rangle\}, \\
h\left(a_{10}^{0}\right) & =\{\langle 1,0\rangle,\langle 3,0\rangle,\langle 1,2\rangle,\langle 3,2\rangle\} .
\end{aligned}
$$

If $\alpha \geq 2$, then $\mathfrak{A}_{\alpha} \notin \mathrm{RA}$, since

$$
\begin{aligned}
& a_{01}^{0} ; a_{10}^{1} ; a_{00}=a_{00} ; a_{00}=a_{0}, \\
& a_{01}^{0} ;\left(a_{10}^{1} ; a_{00}\right)=a_{01}^{0} ;\left(\sum_{\kappa<\alpha} a_{10}^{\kappa}\right)=a_{0}+a_{00} .
\end{aligned}
$$


Let $x=a_{0}$ and $y=a_{1}$. Then Tw $\mathfrak{A}_{\alpha}=\{x, y\}, 1^{\prime}=x+y$, and $x ; 1 ; y=$ $\sum_{\kappa<\alpha} a_{01}^{\kappa}$, while Fn $\mathfrak{A}_{\alpha}=\left\{a_{0}, a_{00}, a_{1}, a_{11}\right\}$.

\section{POINT-DENSITY AND PAIR-DENSITY}

Definition 44. Let $\mathfrak{A} \in N A . \mathfrak{A}$ is point-dense if $\sum \operatorname{Pt} \mathfrak{A}=1$, and $\mathfrak{A}$ is pairdense if $\sum \operatorname{Pr} \mathfrak{A}=1$ '.

It is easy to show (as in the proof of Theorem 47 below) that $\mathfrak{A} \in N A$ is point-dense (or pair-dense) just in case every nonzero identity element contains a point (or pair), i.e., the points (or pairs) are dense below 1'. That is why "dense" is used in Definition 44.

Theorem 45. Every point-dense NA is also pair-dense.

Proof. This follows immediately from Theorem 39(i)(ii).

Theorem 46. Every point-dense SA is a RA.

Proof. Assume $\mathfrak{A} \in \mathrm{SA}$ and $\mathfrak{A}$ is point-dense. The associative law can be proved from the following statement by means of Theorem $8(\mathrm{ix})(\mathrm{xiv})(\mathrm{xv})$ :

$$
x ; y ; z \leq x ;(y ; z) \text { for all } x, y, z \in A .
$$

So we need only prove (1). Given $x, y, z \in A$, let $w=(x ;(y ; z))^{-} \cdot x ; y ; z$. By the IL, point-density, and the complete additivity of ; , we have

$$
w=w ; 1^{\prime}=w ; \sum \operatorname{Pt} \mathfrak{A}=\sum_{v \in \operatorname{Pt} \mathfrak{A}} w ; v,
$$

but, for every $v \in \operatorname{Pt} \mathfrak{A}, v ; 1 ; v=v \leq 1$ ' by Theorem 8 (xxvi), so

$$
\begin{array}{rlrl}
w ; v & =\left((x ;(y ; z))^{-} \cdot x ; y ; z\right) ; v & \\
& =(x ;(y ; z))^{-} ; v \cdot x ; y ; z ; v & & v \leq 1 \text {, Theorem 8(xvii) } \\
& =(x ;(y ; z))^{-} ; v \cdot x ; y ; z ;(v ; 1 ; v) & v=v ; 1 ; v \\
& =(x ;(y ; z))^{-} ; v \cdot x ;(y ; z) ;(v ; 1 ; v) & & \text { Theorem } 25 \\
& =(x ;(y ; z))^{-} ; v \cdot x ;(y ; z) ; v & & v ; 1 ; v=v \\
& =\left((x ;(y ; z))^{-} \cdot x ;(y ; z)\right) ; v & & v \leq 1 \text {, Theorem 8(xvii) } \\
& =0 ; v=0, & &
\end{array}
$$

so $w=0$. Thus (1) holds.

If $\alpha \geq 2$, then the algebra $\mathfrak{A}_{\alpha}$ given in the proof of Theorem 43(ii) is a pair-dense SA which is not a relation algebra.

Theorem 47. Suppose $\mathfrak{A} \in \mathrm{SA}$ and $\mathfrak{A}$ is simple.

(i) If $\mathfrak{A}$ is point-dense, then Ia $\mathfrak{A}=\operatorname{Pt} \mathfrak{A}$.

(ii) If $\mathfrak{A}$ is pair-dense, then Ia $\mathfrak{A}=\operatorname{Pt} \mathfrak{A} \cup \mathrm{Tw} \mathfrak{A}$. 
Proof. (i): By Theorem 40(iii), Pt $\mathfrak{A} \subseteq$ Ia $\mathfrak{A}$. To prove the opposite inclusion, suppose $x \in \operatorname{Ia} \mathfrak{A}$. Then $0 \neq x=x \cdot 1^{\prime}=x \cdot \sum \operatorname{Pt} \mathfrak{A}=\sum_{v \in \operatorname{Pt} \mathfrak{A}} x \cdot v$, so there is some $v \in \operatorname{Pt} \mathfrak{A}$ such that $0 \neq v \cdot x$. But $x \in \operatorname{At} \mathfrak{A}$ and $v \in$ At $\mathfrak{A}$, so $x=v \in \mathrm{Pt} \mathfrak{A}$.

(ii): By Theorem 40 (iii), we need only show Ia $\mathfrak{A} \subseteq \operatorname{Pr} \mathfrak{A}$. Let $x \in$ Ia $\mathfrak{A}$. By pair-density, $0 \neq x=\sum_{v \in \operatorname{Pr} \mathfrak{A}} x \cdot v$, so $x \leq v$ for some $v \in \operatorname{Pr} \mathfrak{A}$. Then $x ; 0^{\prime} ; x ; 0^{\prime} ; x \leq v ; 0^{\prime} ; v ; 0^{\prime} ; v \leq 1^{\prime}$, so $x \in \operatorname{Pr} \mathfrak{A}$.

Theorem 48. Every simple pair-dense RA, and hence every simple point-dense $\mathrm{SA}$, is atomic.

Proof. Assume $\mathfrak{A}$ is a simple pair-dense RA. We will show that every nonzero element of $\mathfrak{A}$ contains at atom. Accordingly, assume $0 \neq x \in A$. Then $x^{\mathrm{d}} \neq 0$ since $x=x^{\mathrm{d}} ; x$. By pair-density, there is some $y \in \operatorname{Pr} \mathfrak{A}$ such that $y \leq x^{\mathrm{d}}$. Then $y=y \cdot x^{\mathrm{d}}=y ; x^{\mathrm{d}} \leq y ; x ; 1$, so $y ; x \neq 0$, which implies $(y ; x)^{r} \neq 0$. By a similar argument, there is some $z \in \operatorname{Pr} \mathfrak{A}$ such that $z \leq(y ; x)^{r}$ and $y ; x ; z \neq 0$.

By Theorem 40(ii)(iii), $y$ is either a point, a twin, or the join of two points, and the same is true of $z$. For each of the resulting nine cases we will show that $y ; 1 ; z$ is either an atom, the join of two atoms, or the join of four atoms.

If $y, z \in \mathrm{Tw} \mathfrak{A}$, then $y ; 1 ; z$ is an atom or the join of two functional atoms by Theorem 43(i).

Suppose $y \in \operatorname{Pt} \mathfrak{A} \cup \operatorname{Tw} \mathfrak{A}$. If $z \in \operatorname{Pt} \mathfrak{A}$, then $y ; 1 ; z \in$ At $\mathfrak{A} \cap$ Fn $\mathfrak{A}$ by Theorem $40(\mathrm{v})$. If $z$ is the join of two points $u$ and $v$, then $y ; 1 ; z=$ $y ; 1 ; u+y ; 1 ; v$, and $y ; 1 ; u, y ; 1 ; v \in$ At $\mathfrak{A} \cap$ Fn $\mathfrak{A}$ by Theorem 40(v), so $y ; 1 ; z$ is the join of two functional atoms. Similarly, $y ; 1 ; z$ is an atom (whose converse is functional) or the join of two atoms (whose converses are functional) if $z \in \operatorname{Pt} \mathfrak{A} \cup \mathrm{Tw} \mathfrak{A}$ and $y \in \operatorname{Pt} \mathfrak{A}$ or $y$ is the join of two points.

Finally, if both $y$ and $z$ are joins of two points, then $y ; 1 ; z$ is the join of four functional atoms by Theorem $40(\mathrm{v})$.

We have $0 \neq y ; x ; z \leq y ; 1 ; z$, and $y ; 1 ; z$ is the join of finitely many atoms, so $y ; x ; z$ must contain one of those atoms. But $y ; x ; z \leq x$, so $x$ also contains at atom.

Now we turn to a key result which says, in effect, that every partial representation of a simple pair-dense RA can be extended wherever necessary. First we need a lemma.

Lemma 49. Assume $2 \leq \alpha, \mathfrak{A} \in \mathrm{WA}$, and $x \in \mathrm{At} \mathfrak{A}$. Then there is some $m \in B_{\alpha} \mathfrak{A}$ such that $m_{01}=x$.

Proof. Define $m: \alpha \times \alpha \rightarrow A$ as follows:

$$
m_{\kappa \lambda}= \begin{cases}x & \text { if } \kappa=0, \lambda \geq 1, \\ \breve{x} & \text { if } \kappa \geq 1, \lambda=0, \\ x^{\mathrm{d}} & \text { if } \kappa=\lambda=0, \\ x^{r} & \text { if } \kappa \geq 1, \lambda \geq 1 .\end{cases}
$$


By Theorem 10(i) and Theorem 12(i), $\breve{x}, x^{\mathrm{d}}, x^{r} \in$ At $\mathfrak{A}$, so $m: \alpha \times \alpha \rightarrow$ At $\mathfrak{A}$. It is now easy to verify that conditions $\left(B_{0}\right)-\left(B_{2}\right)$ hold, using various parts of Theorem 8, so $m \in B_{\alpha} \mathfrak{A}$.

Theorem 50. Let $\mathfrak{A}$ be a simple pair-dense RA. Then $B_{\omega} \mathfrak{A}$ is an $\omega$-dimensional relational basis for $\mathfrak{A}$.

Proof. Condition $\left(\mathrm{R}_{0}\right)$ is trivially satisfied, and $\left(\mathrm{R}_{1}\right)$ holds by Lemma 49 , so it remains to show the extension condition $\left(\mathrm{R}_{2}\right)$ holds. Assume $m \in B_{\omega} \mathfrak{A}$, $\kappa, \lambda, \mu \leq \omega, \mu \neq \kappa, \lambda, x, y \in$ At $\mathfrak{A}$, and $m_{\kappa \lambda} \leq x ; y$.

We begin by defining $m^{\prime}$ for almost all arguments; the exceptions are $m_{\mu \nu}^{\prime}$ and $m_{\nu \mu}^{\prime}$ with $\nu \in \omega \sim\{\kappa, \lambda, \mu\}$.

$$
\begin{gathered}
m_{\mu \mu}^{\prime}=x^{r}=1 \cdot 1 ; x=y^{d}=1 \cdot y ; 1, \\
m_{\kappa \mu}^{\prime}=x, \quad m_{\mu \kappa}^{\prime}=\breve{x}, \quad m_{\mu \lambda}^{\prime}=y, \quad m_{\lambda \mu}^{\prime}=\breve{y}, \\
m_{\nu \xi}^{\prime}=m_{\nu \xi} \quad \text { if } \nu, \xi \in \omega \sim\{\mu\} .
\end{gathered}
$$

The last three equations of (1) hold by Theorem 8 (xxi)(xxii) and Theorem 12(iv). All the elements of $A$ appearing in (1)-(3) are atoms, by Theorems 10(i), 12(i). Two of the desired properties of $m^{\prime}$ are guaranteed by (2), namely $m_{\kappa \mu}^{\prime}=x$ and $m_{\mu \lambda}^{\prime}=y$, and, by (3), $m$ and $m^{\prime}$ will agree up to $\mu$, no matter how the definition of $m^{\prime}$ is completed. What therefore remains is to define $m_{\mu \nu}^{\prime}$ and $m_{\nu \mu}^{\prime}$ for all $\nu \in \omega \sim\{\kappa, \lambda, \mu\}$, and show that $m^{\prime} \in B_{\omega} \mathfrak{A}$.

Let $\Delta_{0}=\{\kappa, \lambda, \mu\}$. For every $\alpha<\omega$, let $\Delta_{\alpha+1}=\Delta_{\alpha} \cup\{\nu\}$, where $\nu$ is the least element of $\omega \sim \Delta_{\alpha}$, and let

$$
\Sigma_{\alpha}=\left(\{\mu\} \times \Delta_{\alpha}\right) \cup\left(\Delta_{\alpha} \times\{\mu\}\right) \cup((\omega \sim\{\mu\}) \times(\omega \sim\{\mu\})) .
$$

Clearly $\Sigma_{\alpha}$ is symmetric and reflexive, i.e., if $\langle\nu, \xi\rangle \in \Sigma_{\alpha}$ then $\langle\nu, \nu\rangle,\langle\xi, \nu\rangle$, $\langle\xi, \xi\rangle \in \Sigma_{\alpha}$, and $\omega \times \omega=\bigcup_{\alpha<\omega} \Sigma_{\alpha}$. Conditions (1)-(3) define $m_{\nu \xi}^{\prime}$ whenever $\langle\nu, \xi\rangle \in \Sigma_{0}$. Using various parts of Theorem 8 , it is easy to show that the following three conditions hold for $\alpha=0$ :

$$
\begin{array}{cl}
m_{\nu \nu}^{\prime} \leq 1, & \text { whenever }\langle\nu, \nu\rangle \in \Sigma_{\alpha}, \\
\breve{m}_{\nu \xi}^{\prime}=m_{\xi \nu}^{\prime} & \text { whenever }\langle\nu, \xi\rangle \in \Sigma_{\alpha}, \\
m_{\nu \xi}^{\prime} \leq m_{\nu \rho}^{\prime} ; m_{\rho \xi}^{\prime} & \text { whenever }\langle\nu, \xi\rangle,\langle\nu, \rho\rangle,\langle\rho, \xi\rangle \in \Sigma_{\alpha} .
\end{array}
$$

Suppose, given some fixed $\alpha<\omega$, that $m_{\mu \nu}^{\prime}$ and $m_{\nu \mu}^{\prime}$ have been defined for every $\nu \in \Delta_{\alpha}$ in such a way that (4)-(6) hold. We will choose $m_{\mu \beta}^{\prime}$ and $m_{\beta \mu}^{\prime}$, where $\{\beta\}=\Delta_{\alpha+1} \sim \Delta_{\alpha}$, and prove that (4)-(6) still hold if $\alpha$ is replaced by $\alpha+1$.

There are four cases, in three of which the definitions of $m_{\mu \beta}^{\prime}$ and $m_{\beta \mu}^{\prime}$ are forced, while in the fourth case $m_{\mu \beta}^{\prime}$ may be either one of two functional atoms. 
For the first case, assume there is some $\nu \in \Delta_{\alpha} \sim\{\mu\}$ such that $m_{\nu \beta}^{\prime} \in \mathrm{Fn} \mathfrak{A}$. Then, by (6),

$$
0 \neq m_{\nu \nu}^{\prime} \leq m_{\nu \beta}^{\prime} ; m_{\beta \nu}^{\prime} \cdot m_{\nu \mu}^{\prime} ; m_{\mu \nu}^{\prime} \leq m_{\nu \beta}^{\prime} ; 1 \cdot m_{\nu \mu}^{\prime} ; 1 .
$$

Using the cycle law and (5), we get

$$
0 \neq m_{\mu \nu}^{\prime} ;\left(m_{\nu \beta}^{\prime} ; 1\right)=m_{\mu \nu}^{\prime} ; m_{\nu \beta}^{\prime} ; 1 .
$$

Consequently $0 \neq m_{\mu \nu}^{\prime} ; m_{\nu \beta}^{\prime}$ by Theorem 6(ii). Thus we have $m_{\mu \nu}^{\prime} \in$ At $\mathfrak{A}$, $m_{\nu \beta}^{\prime} \in \mathrm{Fn} \mathfrak{A}$, and $0 \neq m_{\mu \nu}^{\prime} ; m_{\nu \beta}^{\prime}$, so, by Theorem 4.6(vii) of [JT52],

$$
m_{\mu \nu}^{\prime} ; m_{\nu \beta}^{\prime} \in \text { At } \mathfrak{A}
$$

In this case we define $m_{\mu \beta}^{\prime}$ and $m_{\beta \mu}^{\prime}$ as follows:

$$
m_{\mu \beta}^{\prime}=m_{\mu \nu}^{\prime} ; m_{\nu \beta}^{\prime} \quad \text { and } \quad m_{\beta \mu}^{\prime}=\breve{m}_{\mu \beta}^{\prime} .
$$

For the case under consideration we now establish (6) for $\alpha+1$. Let $\xi \in \Delta_{\alpha+1}$. Then

$$
\begin{aligned}
0 \neq m_{\mu \xi}^{\prime} & =m_{\mu \xi}^{\prime} \cdot m_{\mu \nu}^{\prime} ; m_{\nu \xi}^{\prime} & & (6) \text { if } \xi \neq \beta,(8) \text { if } \xi=\beta \\
& \leq m_{\mu \xi}^{\prime} \cdot m_{\mu \nu}^{\prime} ;\left(m_{\nu \beta}^{\prime} ; m_{\beta \xi}^{\prime}\right) & & (6), \text { Theorem 8(iii) } \\
& =m_{\mu \xi}^{\prime} \cdot m_{\mu \nu}^{\prime} ; m_{\nu \beta}^{\prime} ; m_{\beta \xi}^{\prime} & & \mathrm{AL} \\
& =m_{\mu \xi}^{\prime} \cdot m_{\mu \beta}^{\prime} ; m_{\beta \xi}^{\prime} & & \text { (8). }
\end{aligned}
$$

By the cycle law and (5), $0 \neq m_{\mu \beta}^{\prime} \cdot m_{\mu \xi}^{\prime} ; m_{\xi \beta}^{\prime}$, and hence $m_{\mu \beta}^{\prime} \leq m_{\mu \xi}^{\prime} ; m_{\xi \beta}^{\prime}$ since $m_{\mu \beta}^{\prime} \in$ At $\mathfrak{A}$. Thus we have shown

$$
m_{\mu \beta}^{\prime} \leq m_{\mu \xi}^{\prime} ; m_{\xi \beta}^{\prime} \quad \text { whenever } \xi \in \Delta_{\alpha+1} .
$$

In proving that (4)-(6) hold for $\alpha+1$, we need only consider cases not covered by the assumption that (4)-(6) hold for $\alpha$. There are no such additional cases for (4), the additional cases for (5) follow immediately from (8), and all the additional cases for (6) can be easily derived from (9) using Theorem 8, CL, (5), and (8).

In the second case, we assume $m_{\nu \mu}^{\prime} \in$ Fn $\mathfrak{A}$ for some $\nu \in \Delta_{\alpha} \sim\{\mu\}$. As in the first case, we have $m_{\beta \nu}^{\prime} ; m_{\nu \mu}^{\prime} \in$ At $\mathfrak{A}$. Hence we let $m_{\beta \mu}^{\prime}=m_{\beta \nu}^{\prime} ; m_{\nu \mu}^{\prime}$, $m_{\mu \beta}^{\prime}=\breve{m}_{\beta \mu}^{\prime}$, and show that (4)-(6) hold for $\alpha+1$.

For the last two cases we assume

$$
m_{\nu \mu}^{\prime} \notin \mathrm{Fn} \mathfrak{A} \quad \text { and } \quad m_{\nu \beta}^{\prime} \notin \mathrm{Fn} \mathfrak{A} \quad \text { for every } \nu \in \Delta_{\alpha} \sim\{\mu\} .
$$


Let $\nu \in \Delta_{\alpha} \sim\{\mu\}$. We have $m_{\nu \nu}^{\prime}, m_{\beta \beta}^{\prime} \in$ Ia $\mathfrak{A}$, so, by Theorem 47(ii), $m_{\nu \nu}^{\prime}, m_{\beta \beta}^{\prime} \in \operatorname{Pt} \mathfrak{A} \cup \mathrm{Tw} \mathfrak{A}$. According to Theorems $40(\mathrm{v})$ and $43(\mathrm{i})$, the only way $m_{\nu \nu}^{\prime} ; 1 ; m_{\beta \beta}^{\prime}$ can fail to be an atom is for it to be the join of two functional atoms, which can occur only if $m_{\nu \nu}^{\prime}, m_{\beta \beta}^{\prime} \in \mathrm{Tw} \mathfrak{A}$. But

$$
m_{\nu \beta}^{\prime} \leq m_{\nu \nu}^{\prime} ; m_{\nu \beta}^{\prime} ; m_{\beta \beta}^{\prime} \leq m_{\nu \nu}^{\prime} ; 1 ; m_{\beta \beta}^{\prime}
$$

and $m_{\nu \beta}^{\prime} \in$ At $\mathfrak{A}$, so if $m_{\nu \nu}^{\prime} ; 1 ; m_{\beta \beta}^{\prime}$ were the join of two functional atoms, then $m_{\nu \beta}^{\prime}$ would have to be one of those two functional atoms, contradicting (10). Therefore $m_{\nu \beta}^{\prime}=m_{\nu \nu}^{\prime} ; 1 ; m_{\beta \beta}^{\prime} \in$ At $\mathfrak{A}$. Furthermore, if $m_{\beta \beta}^{\prime} \in \operatorname{Pt} \mathfrak{A}$, then $m_{\nu \beta}^{\prime}=m_{\nu \nu}^{\prime} ; 1 ; m_{\beta \beta}^{\prime} \in \mathrm{Fn} \mathfrak{A}$ by Theorem $40(\mathrm{v})$, again contradicting (10). Hence $m_{\beta \beta}^{\prime} \in \mathrm{Tw} \mathfrak{A}$. The same observations apply with $\mu$ in place of $\beta$. Thus we have

$$
m_{\nu \beta}^{\prime}=m_{\nu \nu}^{\prime} ; 1 ; m_{\beta \beta}^{\prime} \quad \text { and } \quad m_{\nu \mu}^{\prime}=m_{\nu \nu}^{\prime} ; 1 ; m_{\mu \mu}^{\prime} .
$$

Since $0 \neq m_{\nu \nu}^{\prime}=m_{\nu \nu}^{\prime} ; m_{\nu \nu}^{\prime}$ and $\mathfrak{A}$ is simple, we have $1=1 ; m_{\nu \nu}^{\prime} ; m_{\nu \nu}^{\prime} ; 1$. From (4), (5), and (11) we get

$$
m_{\mu \mu}^{\prime} ; 1 ; m_{\beta \beta}^{\prime}=m_{\mu \mu}^{\prime} ; 1 ; m_{\nu \nu}^{\prime} ; m_{\nu \nu}^{\prime} ; 1 ; m_{\beta \beta}^{\prime}=m_{\mu \nu}^{\prime} ; m_{\nu \beta}^{\prime} .
$$

Thus we have shown $m_{\mu \mu}^{\prime}, m_{\beta \beta}^{\prime} \in \mathrm{Tw} \mathfrak{A}$ and

$$
m_{\mu \mu}^{\prime} ; 1 ; m_{\beta \beta}^{\prime}=m_{\mu \nu}^{\prime} ; m_{\nu \beta}^{\prime} \quad \text { for every } \nu \in \Delta_{\alpha} \sim\{\mu\} .
$$

By Theorem 43(i), $m_{\mu \mu}^{\prime} ; 1 ; m_{\beta \beta}^{\prime}$ is either an atom or the join of two functional atoms. If $m_{\mu \mu}^{\prime} ; 1 ; m_{\beta \beta}^{\prime}$ is an atom, we must let $m_{\mu \beta}^{\prime}=m_{\mu \mu}^{\prime} ; 1 ; m_{\beta \beta}^{\prime}$, but if $m_{\mu \mu}^{\prime} ; 1 ; m_{\beta \beta}^{\prime}$ is not an atom, then we may let $m_{\mu \beta}^{\prime}$ be either one of the two functional atoms which $m_{\mu \mu}^{\prime} ; 1 ; m_{\beta \beta}^{\prime}$ contains. Both cases can be handled simultaneously by assuming only

$$
m_{\mu \beta}^{\prime}=z \in \text { At } \mathfrak{A}, \quad z \leq m_{\mu \mu}^{\prime} ; 1 ; m_{\beta \beta}^{\prime}, \quad \text { and } \quad m_{\beta \mu}^{\prime}=\breve{m}_{\mu \beta}^{\prime} .
$$

From (13) we get $\left(m_{\mu \beta}^{\prime}\right)^{d}=m_{\mu \mu}^{\prime}$ by Theorems $12(\mathrm{v})$ and $8(\mathrm{xxv})(\mathrm{iii})$, so $m_{\mu \beta}^{\prime}=$ $\left(m_{\mu \beta}^{\prime}\right)^{\mathrm{d}} ; m_{\mu \beta}^{\prime}=m_{\mu \mu}^{\prime} ; m_{\mu \beta}^{\prime}$ by Theorem 8(xxiv), and, similarly, $m_{\mu \beta}^{\prime}=$ $m_{\mu \beta}^{\prime} ; m_{\beta \beta}^{\prime}$. These two cases, together with those following from (12) and $m_{\mu \beta}^{\prime} \leq m_{\mu \mu}^{\prime} ; 1 ; m_{\beta \beta}^{\prime}$, give us

$$
m_{\mu \beta}^{\prime} \leq m_{\mu \nu}^{\prime} ; m_{\nu \beta}^{\prime} \quad \text { for every } \nu \in \Delta_{\alpha+1} .
$$

It follows from (13) and (14) that (4)-(6) hold for $\alpha+1$.

This completes a proof by induction that $m^{\prime}$ can be constructed so that (4)(6) hold for every $\alpha<\omega$. Since $\omega \times \omega=\bigcup_{\alpha<\omega} \Sigma_{\alpha}$, it follows that $m^{\prime} \in$ $B_{\omega} \mathfrak{A}$. 


\section{REPRESENTATION RESULTS}

In the first two results we restrict our attention to simple algebras.

Theorem 51. Let $\mathfrak{A}$ be a simple pair-dense RA. For every set $U$ the following statements are equivalent:

( $\alpha) \mathfrak{A}$ has a complete representation over $U$,

(B) $|U|=|\mathrm{Pt} \mathfrak{A}|+2|\mathrm{Tw} \mathfrak{A}|$.

Proof. First we show that $(\alpha)$ implies $(\beta)$. Suppose that $R$ is a complete representation of $\mathfrak{A}$ over $U$. For every $x \in \operatorname{Pt} \mathfrak{A}, R(x)$ is a point of $\mathfrak{R e} U$, and so there is some $a \in U$ such that $R(x)=\left\{\left\langle a, a_{\rangle}\right\}\right.$. Distinct points of $\mathfrak{A}$ must correspond to distinct elements of $U$, since $R$ is one-to-one. This establishes a one-to-one correspondence between $\mathrm{Pt} \mathfrak{A}$ and a subset of $U$. Now let $y \in \operatorname{Tw} \mathfrak{A} . R(y)$ is not a twin of $\mathfrak{R e} U$ (in fact, Tw $\mathfrak{R e} U=\varnothing$ ), but $R(y$ ) is a pair of $\mathfrak{R e} U$ which is not a point. Hence there are distinct $b, c \in U$ such that $R(y)=\{\langle b, b\rangle,\langle c, c\rangle\}$. Thus every pair of $\mathfrak{A}$ corresponds to a two-element subset of $U$. Distinct identity atoms are disjoint, so distinct pairs in $\mathfrak{A}$ correspond to disjoint two-element subsets of $U$, and an element of $U$ corresponding to a point of $\mathfrak{A}$ cannot also correspond to a pair. Thus there is a one-to-one correspondence between $\mathrm{Tw} \mathfrak{A}$ and a collection of two-element subsets of $U$, each disjoint from the subset corresponding to $\mathrm{Pt} \mathfrak{A}$. It follows that $|U| \geq|\operatorname{Pt} \mathfrak{A}|+2 \mid$ Tw $\mathfrak{A} \mid$. To get the inequality in the other direction, it suffices to show every element of $U$ corresponds to some point or pair of $\mathfrak{A}$. Let $a \in U . \mathfrak{A}$ is atomic, so $1^{\prime}=\sum \operatorname{Ia} \mathfrak{A} . R$ is complete, so $\operatorname{Id}_{U}=R\left(1^{\prime}\right)=$ $R\left(\sum \operatorname{Ia} \mathfrak{A}\right)=\bigcup\{R(x): x \in \operatorname{Ia} \mathfrak{A}\}$. Thus $\{\langle a, a\rangle\} \subseteq \operatorname{Id}_{U}=\bigcup\{R(x): x \in \operatorname{Ia} \mathfrak{A}\}$, so there is some $x \in \operatorname{Ia} \mathfrak{A}$ such that $\{\langle a, a\rangle\} \subseteq R(x)$. Every identity atom of $\mathfrak{A}$ is a point or a pair, so either $a$ corresponds to a point, or else $a$ is one of two elements of $U$ corresponding to a pair. This shows $|U| \leq|\operatorname{Pt} \mathfrak{A}|+2|\mathrm{Tw} \mathfrak{A}|$, completing the proof that $(\alpha)$ implies $(\beta)$.

Now assume $|U|=|\operatorname{Pt} \mathfrak{A}|+2 \mid$ Tw $\mathfrak{A} \mid$. Every simple pair-dense RA has an $\omega$ dimensional relational basis by Theorem 50 and is atomic by Theorem 48 . An atomic RA with an $\omega$-dimensional relational basis is representable by Theorem 37. Thus we know $\mathfrak{A}$ is simple, atomic, and representable.

Let $x, y \in$ At $\mathfrak{A}$. Then $x ; y \leq x^{\mathrm{d}} ; 1 ; y^{r}$ and $x^{\mathrm{d}}, y^{\mathrm{r}} \in \mathrm{Ia} \mathfrak{A}$ by Theorems 8(xxiv) and 12(i), so $x^{d}, y^{r} \in \operatorname{Pt} \mathfrak{A} \cup \operatorname{Tw} \mathfrak{A}$ by Theorem 47(ii). By Theorem $40(v)$ and Theorem 43(i), either $x^{d} ; 1 ; y^{r}$ is an atom or $x^{d} ; 1 ; y^{r}$ is the join of two atoms. Thus $\mid\{z: x ; y \geq z \in$ At $\mathfrak{A}\} \mid \leq 2$ for all $x, y \in$ At $\mathfrak{A}$. By Theorem $33, \mathfrak{A}$ is completely representable. Since $\mathfrak{A}$ is also simple, there is a set $V$ and a complete representation $R$ of $\mathfrak{A}$ over $V$. Since $(\alpha)$ implies $(\beta)$, we conclude that $|V|=|\operatorname{Pt} \mathfrak{A}|+2|\mathrm{Tw} \mathfrak{A}|=|U|$. Choose $f: V \rightarrow U$ so that $f$ is a one-to-one correspondence. Define $R^{\prime}: A \rightarrow \operatorname{Sb}(U \times U)$ by $R^{\prime}(x)=f^{-1}|R(x)| f$ for every $x \in A$. It is easy to confirm that $R^{\prime}$ is a complete representation of $\mathfrak{A}$ over $U$. 
Theorem 52. (i) If $\mathfrak{A}$ is a simple point-dense SA, then for every set $U$ the following statements are equivalent:

$(\alpha) \mathfrak{A}$ has a complete representation over $U$;

(B) $|U|=|\mathrm{Pt} \mathfrak{A}|$.

(ii) $\mathfrak{A} \cong \mathfrak{R e} U$ iff $\mathfrak{A}$ is a simple complete point-dense $\mathrm{SA}$ and $|U|=|\mathrm{Pt} \mathfrak{A}|$.

(iii) $\mathfrak{A} \cong \mathfrak{R e} P t \mathfrak{A}$ iff $\mathfrak{A}$ is a simple complete point-dense SA.

Proof. (i): By Theorems 45, 46, 47, and 39(iii), every simple point-dense SA is a simple pair-dense $\mathrm{RA}$ in which $\operatorname{Tw} \mathfrak{A}=\varnothing$. The equivalence of $(\alpha)$ and $(\beta)$ therefore follows from Theorem 51.

(ii): For one direction it suffices to observe that $\mathfrak{R e} U$ is a simple complete point-dense SA and Pt $\mathfrak{R e} U=\{\{\langle a, a\rangle\}: a \in U\}$.

Assume $\mathfrak{A}$ is a simple complete point-dense $S A$ and $|U|=|\mathrm{Pt} \mathfrak{A}|$. By part (i) there is a complete representation $R$ of $\mathfrak{A}$ over $U$. Let $x \in$ At $\mathfrak{A}$. Then $x^{\mathrm{d}}, r^{\mathrm{r}} \in \mathrm{Ia} \mathfrak{A}=\operatorname{Pt} \mathfrak{A}$ by Theorems $12(\mathrm{i})$ and $47(\mathrm{i})$, so $x=x^{\mathrm{d}} ; 1 ; x^{\mathrm{r}}$, since $x=x^{\mathrm{d}} ; x ; x^{r} \leq x^{\mathrm{d}} ; 1 ; x^{r} \in$ At $\mathfrak{A}$ by Theorem $40(\mathrm{v})$. Also, $R\left(x^{\mathrm{d}}\right), R\left(x^{r}\right) \in$ Pt $\Re \mathfrak{R e}$, so there are $a, b \in U$ such that $R\left(x^{\mathrm{d}}\right)=\{\langle a, a\rangle\}, R\left(x^{r}\right)=\{\langle b, b\rangle\}$, and hence

$$
R(x)=R\left(x^{\mathrm{d}} ; 1 ; x^{\mathrm{r}}\right)=\{\langle a, a\rangle\}|(U \times U)|\{\langle b, b\rangle\}=\{\langle a, b\rangle\} .
$$

Thus $R$ maps At $\mathfrak{A}$ into At $\mathfrak{R e} U$.

Note that $\mathfrak{A}$ is atomic by Theorem 48 , so $1=\sum$ At $\mathfrak{A} . R$ is complete, so $U \times U=R(1)=R\left(\sum\right.$ At $\left.\mathfrak{A}\right)=\bigcup\{R(x): x \in$ At $\mathfrak{A}\}$. Hence every atom $\{\langle a, b\rangle\}$ of $\mathfrak{R e} U$ is the image of some atom of $\mathfrak{A}$.

Thus the embedding $R$ establishes a one-to-one correspondence between the atoms of $\mathfrak{A}$ and $\mathfrak{R e} U$. Since $R$ is complete, $R$ must be an isomorphism.

(iii): This part follows from part (ii).

It is possible to construct a somewhat more direct proof of Theorem 52(iii). Define a function $R: A \rightarrow \operatorname{Sb}(\operatorname{Pt} \mathfrak{A} \times \mathrm{Pt} \mathfrak{A})$ by

$$
R(x)=\{\langle u, v\rangle: u, v \in \operatorname{Pt} \mathfrak{A} \text { and } 1 ; u ; x ; v ; 1=1\}
$$

for every $x \in A$. It can then be shown that $R$ is a complete representation of $\mathfrak{A}$ over Pt $\mathfrak{A}$. The author's original (unpublished, 1973) proof of Theorem 52(iii) proceeded in this way.

In the final theorem we consider algebras which may not be simple. Here we encounter the difficulty that pair-density and point-density are not preserved by homomorphisms. In fact, we can construct an example which shows that a point-dense RA may have a simple homomorphic image which is not even pair-dense.

Let $\mathfrak{M}_{3}$ be the subalgebra of $\mathfrak{R e} 3$ which has universe $M_{3}=\left\{\varnothing, \mathrm{Id}_{3}, \mathrm{Di}_{3}\right.$, $3 \times 3\}$. Then $\mathfrak{M}_{3}$ is neither pair-dense nor point-dense. (The subalgebra $\mathfrak{M}_{2}$ of $\mathfrak{R e} 2$ with universe $M_{2}=\left\{\varnothing, \mathrm{Id}_{2}, \mathrm{Di}_{2}, 2 \times 2\right\}$ is also not point-dense, but 
it is pair-dense.) Let

$$
\begin{aligned}
h=\{\langle R, S\rangle: & R \in{ }^{\omega} \mathrm{Sb}(3 \times 3) \\
& \left.\& S \in M_{3} \&(\exists \kappa<\omega)(\forall \lambda)\left(\kappa<\lambda<\omega \rightarrow R_{\lambda}=S\right)\right\} .
\end{aligned}
$$

The domain of $h$ consists of all those "eventually constant" sequences whose "limits" are in $M_{3}$, and $h$ maps each such sequence to its limit. Let $A=$ Dom $h$. It is easy to check that $A$ is the universe of a subalgebra $\mathfrak{A}$ of ${ }^{\omega} \mathfrak{R e} 3$. The points of $\mathfrak{A}$ are those sequences which have either $\{\langle 0,0\rangle\},\{\langle 1,1\rangle\}$, or $\{\langle 2,2\rangle\}$ in only finitely many places, and $\varnothing$ everywhere else. Any identity element of $\mathfrak{A}$ contains a point, so $\mathfrak{A}$ is point-dense. Finally, $h$ is a homomorphism mapping $\mathfrak{A}$ onto $\mathfrak{M}_{3}$.

Note that $\mathfrak{A}$ is incomplete. For complete algebras the situation is different, as the next lemma shows.

Lemma 53. Suppose $\mathfrak{A}$ is a complete pair-dense SA.

(i) If $0 \neq x \leq 1$, then there is some $y \in \operatorname{Pr} \mathfrak{A}$ such that $y \leq x$ and $1 ; y ; 1=1 ; x ; 1$.

(ii) Every homomorphic image of $\mathfrak{A}$ is pair-dense.

Proof. (i): Let $\Phi$ be the family of sets $Y$ having these properties:

(1) $Y \subseteq \operatorname{Pr} \mathfrak{A}$

(2) if $u \in Y$ then $u \leq x$,

(3) if $u, v \in Y$ and $u \neq v$, then $1 ; u ; 1 \cdot 1 ; v ; 1=0$.

Note that $\Phi$ is closed under unions of chains. Let $Y$ be a maximal set in $\Phi$. Set $y=\sum Y$, which exists since $\mathfrak{A}$ is complete. We have $y \leq x$ by (2), and so $1 ; y ; 1 \leq 1 ; x ; 1$. To prove the opposite inequality, assume $(1 ; y ; 1)^{-} \cdot x \neq 0$. By pair-density there is some $u \in \operatorname{Pr} \mathfrak{A}$ such that $u \leq(1 ; y ; 1)^{-} \cdot x$. Let $Y^{\prime}=Y \cup\{u\}$. We claim $Y^{\prime} \in \Phi$. Since $u \in \operatorname{Pr} \mathfrak{A}$ and $u \leq x$, we need only check property (3) for $Y^{\prime}$. For every $v \in Y$ we have $1 ; v ; 1 \leq 1 ; y ; 1$, and therefore $1 ; v ; 1 \cdot 1 ; u ; 1 \leq 1 ; y ; 1 \cdot(1 ; y ; 1)^{-}=0$. This not only confirms (3), but also shows that $u \notin Y$, thus contradicting the maximality of $Y$. We may conclude that $(1 ; y ; 1)^{-} \cdot x=0$, i.e., $x \leq 1 ; y ; 1$, which implies $1 ; x ; 1 \leq 1 ; y ; 1$. Thus $1 ; x ; 1=1 ; y ; 1$. Since $x \neq 0$, this also gives $y \neq 0$.

What remains is to show that $y \in \operatorname{Pr} \mathfrak{A}$. First note that

$$
y ; 0^{\prime} ; y ; 0^{\prime} ; y=\sum Y ; 0^{\prime} ; \sum Y ; 0^{\prime} ; \sum Y=\sum_{u, v, w \in Y} u ; 0^{\prime} ; v ; 0^{\prime} ; w \text {. }
$$

Suppose $u, v, w \in Y$. If $u \neq v$ then $u ; 0^{\prime} ; v ; 0$; $w \leq 1 ; u ; 1 \cdot 1 ; v ; 1=0$, and, similarly, if $v \neq w$ or $u \neq w$ then $u ; 0^{\prime} ; v ; 0^{\prime} ; w=0$. Therefore

$$
y ; 0^{\prime} ; y ; 0^{\prime} ; y=\sum_{u \in Y} u ; 0^{\prime} ; u ; 0^{\prime} ; u \leq 1^{\prime} \text {. }
$$


The last inclusion holds by (1). Since $y \neq 0$, we have $y \in \operatorname{Pr} \mathfrak{A}$.

(ii): Suppose $h$ is a homomorphism from $\mathfrak{A}$ onto $\mathfrak{B}$. Let $z \in B$ and $0 \neq z \leq 1^{\prime}$. Choose $w \in A$ such that $h(w)=z$ and set $x=w \cdot 1$ '. Therefore $h(x)=z$ and $0 \neq x \leq 1^{\prime}$. By part (i) there is some $y \in \operatorname{Pr} \mathfrak{A}$ such that $y \leq x$ and $1 ; x ; 1=1 ; y ; 1$. We will show that $h(y) \leq z$ and $h(y) \in \operatorname{Pr} \mathfrak{B}$.

First, $h(y) \leq h(x)=z$. Next, $1 ; z ; 1=1 ; h(x) ; 1=h(1 ; x ; 1)=$ $h(1 ; y ; 1)=1 ; h(y) ; 1$, but $z \neq 0$, so $h(y) \neq 0$ as well. Finally, $h(y) ; 0$; $h(y) ; 0^{\prime} ; h(y)=h\left(y ; 0^{\prime} ; y ; 0^{\prime} ; y\right) \leq h\left(1^{\prime}\right)=1^{\prime}$, so $h(y) \in \operatorname{Pr} \mathfrak{B}$.

Theorem 54. Every pair-dense RA, and hence every point-dense SA, is completely representable.

Proof. Suppose $\mathfrak{A} \in R A$ and $\mathfrak{A}$ is pair-dense. Let $\mathfrak{B}$ be a completion of $\mathfrak{A}$. We have $\operatorname{Pr} \mathfrak{A} \subseteq \operatorname{Pr} \mathfrak{B}$ since $\mathfrak{A} \subseteq \mathfrak{B}$, so $\sum^{(\mathfrak{B})} \operatorname{Pr} \mathfrak{A} \leq \sum^{(\mathfrak{B})} \operatorname{Pr} \mathfrak{B}$. But $1^{\prime}=\sum^{(\mathfrak{A})} \operatorname{Pr} \mathfrak{A}$ since $\mathfrak{A}$ is pair-dense, and $\sum^{(\mathfrak{A})} \operatorname{Pr} \mathfrak{A}=\sum^{(\mathfrak{B})} \operatorname{Pr} \mathfrak{A}$ since $\mathfrak{B}$ is a completion of $\mathfrak{A}$, so $1^{\prime}=\sum^{(\mathfrak{B})} \operatorname{Pr} \mathfrak{B}$, i.e., $\mathfrak{B}$ is pair-dense.

Every relation algebra is a subdirect product of simple relation algebras. (See Theorem 4.15 of [JT52] or Corollary 4.6 of [J82].) Hence, for some index set $I$, there is an $I$-indexed system $\left\langle\mathfrak{C}_{i}: i \in I\right\rangle$ of relation algebras and an embedding $h$ such that

(1) $h: \mathfrak{B} \rightarrow \prod_{i \in I} \mathfrak{C}_{i}$,

(2) for every $i \in I, \mathfrak{C}_{i}$ is a simple homomorphic image of $\mathfrak{B}$.

Choose an $I$-indexed system of sets $\left\langle U_{i}: i \in I\right\rangle$ so that

(3) $U_{i} \cap U_{j}=\varnothing$ whenever $i, j \in I$ and $i \neq j$,

(4) $\left|U_{i}\right|=\left|\mathrm{Pt} \mathfrak{C}_{i}\right|+2\left|\mathrm{Tw}_{\mathfrak{C}}\right|$ for every $i \in I$.

Suppose $j \in I . \mathfrak{B}$ is pair-dense and complete, so $\mathfrak{C}_{j}$ is also pair-dense by (2) and Lemma 53(ii). Hence, by (4) and Theorem 51, there is a complete representation $R_{j}$ of $\mathfrak{C}_{j}$ over $U_{j}$. Let $p_{j}$ be the natural projection homomorphism from $\prod_{i \in I} \mathfrak{C}_{i}$ onto $\mathfrak{C}_{j}$. Let

$$
E=\bigcup_{i \in I} U_{i} \times U_{i}
$$

Note that $E$ is an equivalence relation by (3). Define a function $R: A \rightarrow \mathrm{Sb} E$ by

$$
R(x)=\bigcup_{i \in I} R_{i}\left(p_{i}(h(x))\right) \quad \text { for all } x \in A .
$$

It is now easy to show that $R$ is a complete representation of $\mathfrak{A}$ over $E$.

\section{REFERENCES}

[CT51] Louise H. Chin and Alfred Tarski, Distributive and modular laws in the arithmetic of relation algebras, Univ. of California Publ. Math. (N.S.) 1 (1951), 341-384.

[D1856] Augustus De Morgan, On the symbols of logic, the theory of the syllogism, and in particular of the copula, and the application of the theory of probabilities to some questions in the theory of evidence, Trans. Cambridge Philos. Soc. 9 (1856), 79-127.

[D1864] _, On the syllogism, no. IV, and on the logic of relations, Trans. Cambridge Philos. Soc. 10 (1864), 331-358. 
[D66] _ - On the syllogism, and other logical writings, edited, with an Introduction by Peter Heath, Yale Univ. Press, New Haven, Conn., 1966, pp. xxxi+355.

[HMT71] Leon Henkin, J. Donald Monk, and Alfred Tarski, Cylindric algebras, Part I, NorthHolland, Amsterdam, 1971.

[HMT71] _ _ Cylindric algebras, Part II, North-Holland, Amsterdam, 1985.

[J82] Bjarni Jónsson, Varieties of relation algebras, Algebra Universalis 15 (1982), 273-298.

[JT48] Bjarni Jónsson and Alfred Tarski, Representation problems for relation algebras, Abstract 89, Bull. Amer. Math. Soc. 54 (1948), pp. 80 and 1192.

[JT51] _ Boolean algebras with operators, Part I, Amer. J. Math. 73 (1951), 891-939.

[JT52] _ Boolean algebras with operators, Part II, Amer. J. Math. 74 (1952), 127-162.

[K55] John L. Kelley, General topology, Van Nostrand, London, 1955. Reprint by Springer-Verlag, New York, 1975.

[L50] Roger C. Lyndon, The representation of relational algebras, Ann. of Math. (2) 51 (1950), 707-729.

[L56] - The representation of relation algebras. II, Ann. of Math. (2) 63 (1956), 294-307.

[L61] _ Relation algebras and projective geometries, Michigan Math. J. 8 (1961), 21-28.

[Ma78] Roger D. Maddux, Some sufficient conditions for the representability of relation algebras, Algebra Universalis 8 (1978), 162-172.

[Ma78a] _ , Topics in relation algebras, Doctoral dissertation, Univ. of California, Berkeley, 1978, pp. iii+241.

[Ma81] _ , The equational theory of $C A_{3}$ is undecidable, J. Symbolic Logic 45 (1980), 311-316.

[Ma82] _ Some varieties containing relation algebras, Trans. Amer. Math. Soc. 272 (1982), 501-526.

[Ma83] _ _ A sequent calculus for relation algebras, Ann. Pure Appl. Logic 25 (1983), 73-101.

[Ma89] __, Nonfinite axiomatizability results for cylindric and relation algebras, J. Symbolic Logic 54 (1989), 951-974.

[MT76] Roger D. Maddux and Alfred Tarski, A sufficient condition for the representability of relation algebras, Notices Amer. Math. Soc. 23 (1976), A-447.

[Me87] Elliott Mendelson, Introduction to mathematical logic, 3rd ed., Wadsworth and Brooks/Cole, Monterey, 1987.

[Mo64] J. Donald Monk, On representable relation algebras, Michigan Math. J. 11 (1964), 207-210.

[Mo70] __ Completions of Boolean algebras with operators, Math. Nachr. 46 (1970), 47-55.

[N85] Istvan Németi, Logic with 3 variables has Gödel's incompleteness property-thus free cylindric algebras are not atomic, Ann. Pure Appl. Logic (submitted).

[N86] _ Free algebras and decidability in algebraic logic, Doctoral dissertation, Hungarian Academy of Sciences, Budapest, 1986.

[P33] Charles Sanders Peirce, Collected papers, Vol. III, edited by Charles Hartshorne and Paul Weiss, Harvard Univ. Press, Cambridge, 1933, pp. xiv+433.

[S1895] F. W. K. Ernst Schröder, Vorlesungen über die Algebra der Logik (exakte Logik), Vol. III, Algebra und Logik der Relative, Part I, Leipzig, 1895, pp. viii+649. Reprint by Chelsea, Bronx, 1966.

[SS85] Gunter Schmidt and Thomas Ströhlein, Relation algebras: concept of points and representability, Discrete Math. 54 (1985), 83-92.

[T41] Alfred Tarski, On the calculus of relations, J. Symbolic Logic 6 (1941), 73-89.

[T53] _ Some metalogical results concerning the calculus of relations, J. Symbolic Logic 18 (1953), 188-189. 
[T53a] _ _ A formalization of set theory without variables, J. Symbolic Logic 18 (1953), 189.

[T55] __ Contributions to the theory of models. III, Nederl. Akad. Wetensch. Proc. Ser. A Math. Sci. 58 (1955), 56-64.

[TG87] Alfred Tarski and Steven Givant, A formalization of set theory without variables, Colloq. Publ., vol. 41, Amer. Math. Soc., Providence, R.I., 1987.

[WR10] Alfred North Whitehead and Bertrand Russell, Principia mathematica, Vol. I, Cambridge Univ. Press, 1910, pp. xv+666.

Department of Mathematics, Iowa State University, Ames, Iowa 50011 